Revue d'histoire de l'Amérique française

ZRS REVUE D.HISTOIRE DE L'AMÉRIQUE FRANÇAISE

\title{
Découvertes portugaises en Amérique du Nord
}

\section{Lucien Campeau}

Volume 20, numéro 2, septembre 1966

URI : https://id.erudit.org/iderudit/302566ar

DOI : https://doi.org/10.7202/302566ar

Aller au sommaire du numéro

Éditeur(s)

Institut d'histoire de l'Amérique française

ISSN

0035-2357 (imprimé)

1492-1383 (numérique)

Découvrir la revue

Citer cet article

Campeau, L. (1966). Découvertes portugaises en Amérique du Nord. Revue

d'histoire de l'Amérique française, 20(2), 171-227.

https://doi.org/10.7202/302566ar d'utilisation que vous pouvez consulter en ligne.

https://apropos.erudit.org/fr/usagers/politique-dutilisation/ 


\section{DÉCOUVERTES PORTUGAISES EN AMERIQUE DU NORD}

La discussion qui va suivre ne pourra pas être appelée une histoire des découvertes portugaises en Amérique du Nord. Car nous ne nous occuperons guère de la suite des événements. Notre but est plutôt de soulever et de résoudre, autant qu'il sera en notre pouvoir, les problèmes les plus graves qui concernent ces découvertes. Bien que le Groenland ne soit pas partie de notre continent, c'est à lui d'abord qu'il faudra nous intéresser, ne serait-ce que pour distinguer sa découverte de celle de TerreNeuve. Nous discuterons ensuite la date de la découverte de Terre-Neuve par Gaspar Corte-Real. Nous essaierons d'évaluer l'étendue des explorations portugaises sur notre littoral et nous tâcherons de situer les explorations de Jean Alvares Fagundes.

\section{REDÉCOUVERTE DU GROENLAND (1500-1502)}

L'épisode de la redécouverte du Groenland a été entouré d'une abondante confusion, à la fois parce que la tradition historique s'en est rapidement corrompue et à cause des efforts qu'on a faits pour en attribuer le mérite à Jean ou à Sébastien Cabot. Nous attendrons désormais qu'on nous prouve que Jean Cabot est venu en Amérique pour nous occuper de lui ${ }^{1}$. Et nous nous en tenons aux sources qui concernent les voyages portugais. Il est vrai que les pays nordiques avaient conservé le souvenir d'une ancienne colonisation scandinave au Groenland. Et le cartographe Ruysch ne paraît pas avoir hésité, en 1507/1508, à rendre son vrai nom à la grande île arctique que les Portugais présentaient sous celui de Labrador ${ }^{2}$. Toutefois, la cartographie

\footnotetext{
1 Nous avons exposé notre pensée sur ce sujet, dans cette même Revue, XIX (1965) : 384-413.

2 Voir reproduction dans Konrad Kretchmer, Die Entdeckung Ameri$k a$ 's in ihrer Bedeutung für die Geschichte des Weltbildes (Berlin-LondonParis, 1892), Tafel IX, $\mathrm{n}^{\circ} 2$.
} 
avait alors son centre en pays latins: le Portugal, à ce moment, prenait la relève de l'Italie. Et la science méditerranéenne avait moins de raisons que celle du nord de se souvenir du Groenland abandonné des Européens. Aussi, l'apparition de cette terre, revue en 1500, fit aux Portugais, et même aux Anglais, l'impression d'une découverte tout à fait originale.

Un premier fait à constater, c'est que le Groenland reparaît à l'ouest de l'Europe à partir de $1500^{3}$. Et du coup, il y demeure, situé à peu près en son vrai lieu géographique, alors que les géographes du passé l'indiquaient avec hésitation au nord de l'Europe. Ce phénomène ne peut guère s'expliquer que par une découverte, à ce moment. Sur cette découverte, on est documenté, non pas abondamment, mais catégoriquement.

Le plus ancien témoignage attestant le fait de la découverte est sans doute celui de Pietro Pasqualigo, écrivant de Lisbonne le 18 octobre 1501. Signalant le retour dans la capitale portugaise d'une caravelle en provenance de Terre-Neuve, pays encore sans nom, mais qui est bien identifié, Pasqualigo remarquait en passant:

Les gens de la caravelle croient que la susdite terre (Terre-Neuve) est un continent et qu'elle est unie avec une autre terre, qui a été découverte l'an passé sous le pôle nord par les autres caravelles de cette Majesté, bien qu'elles n'aient pu arriver jusqu'à elle, parce que la mer y était toute glacée de grandes quantités de neige en forme de montagnes ${ }^{4}$.

Il est difficile d'imaginer que ce texte puisse faire difficulté. Il est évident que les "autres caravelles" qui ont décou-

\footnotetext{
${ }^{3}$ La première carte à la représenter d'une manière reconnaissable est celle de Cantino, de 1502.

4 "Credeno questi di la caravella la soprascrita terra esser terra ferma, et conjungerse con altra terra, laqual l'anno' passato soto la tramontana fu discoperta da l'altre caravele de questa Maiestà. Licet non potesseno arrivar a quella per esser et mar li agiazato con grandissima quantità di neve in modo che monti" (Biggar, Les Précurseurs de Jacques Cartier, 1497-1534 (Ottawa, 1913), 65). Le même Pasqualigo, écrivant à ses frères, le 19 octobre, parlait de la même découverte: "laqual (Terre-Neuve) continue in un altra terra che l'anno passato fo discoperta sotto la tramontana, lequal caravelle non posseno arrivar fin là per esser il mar agliazato et infinita copia de neve" (Henry Harrisse, Les Corte-Real et leurs voyages au. Nouveau-Monde (Paris, 1883), 211).
} 
vert "l'an passé" (1500) une autre terre que Terre Neuve "sous le pôle nord" ne sont pas les caravelles qui, en 1501, ont découvert Terre-Neuve. Si l'on assigne à Gaspar Corte-Real, et à juste titre, le commandement des caravelles qui vont à TerreNeuve en 1501, selon Cantino et Pasqualigo ${ }^{5}$, rien ne justifie de lui donner aussi le commandement des caravelles qui ont voyagé précédemment plus au nord. D'après les mêmes Cantino et Pasqualigo, les découvreurs de Terre-Neuve n'ont vu aucune autre terre entre leur départ de Lisbonne et leur arrivée dans la grande île canadienne. Quant à ceux qui se sont approchés d'une terre plus septentrionale sans pouvoir y aborder à cause des glaces, ce sont les découvreurs de 1500: le mot licet, bien que, relie cette circonstance à la découverte faite sous le pôle, c'est-à-dire au Groenland.

Cela est bien certain. Voici comment Cantino, le premier cartographe à présenter au monde le nouveau Groenland, en raconte la découverte:

Cette terre a été découverte sur l'ordre du très haut et très excellent prince-roi, le roi dom Manuell, roi de Portugal. On croit qu'elle est la pointe de l'Asie. Et ceux qui la découvrirent n'abordèrent pas à terre, mais ils la virent [seulement]. Et ils ne virent que des montagnes très denses. C'est pourquoi, selon l'opinion des cosmographes, on croit qu'elle est la pointe de l'Asie ${ }^{6}$.

Et une autre carte portugaise, postérieure à 1520, rapporte un témoignage semblable:

Des Portugais ont découvert cette terre, mais ils n'y entrèrent pas ?.

5 Pasqualigo, écrivant à ses frères le 19 octobre, mentionne expressément le nom de "Gaspar Corterat" (Harrisse, op. cit., 211).

6 "Esta terra he descoberta per mandado do muy alto excelentissimo principe Rey dom manuell Rey de portugall a qual se cree ser esta a ponta dasia $\mathrm{E}$ os que a descobriram nom chegarõ a terra mais vironla \& nam viron senam serras muyto espessas polla quall segum a opinione dos cosmofricos (sic) se cree ser a ponta dasia" (Carte Cantino, ca 1502, dans Cortesão et Teixeira da Mota, Portugaliz Monumenta cartographica (6 vol., Lisboa, 1960), I: estampas 4 et 5).

7 "Terram istam portugalenses invener[unt] atamen non intraverunt" (Carte Munich-Portugaise, apr. 1520, dans Cortesão, op. cit., I: estampa 12). 
Cette circonstance de la redécouverte du Groenland a laissé une trace assez curieuse dans la toponymie de ce pays sur quelques cartes très anciennes. La carte Kunstmann III est celle qui en donne la version la plus reconnaissable: "c [abo] de mirame et Lexame" (cap de regarde-moi et laisse-moi) ${ }^{8}$. Deux autres cartes donnent une forme corrompue et disjointe du même nom: la Kunstmann II ${ }^{9}$, qui a damirla (da mirarla, de la regarder) et de farlla (pour dejarlà, la laisser), et une carte du British Museum (Add. MS 31316) ${ }^{10}$, qui a da mirla (da mirarla) et de sarla (dejarla). Il y a sûrement une relation entre ce nom étrange et le fait qu'on ne put descendre au Groenland la première fois qu'on l'aperçut.

La redécouverte du Groenland est donc identifiée par une circonstance bien particulière: les découvreurs approchèrent suffisamment du littoral pour en considérer l'aspect, mais ils ne purent débarquer à cause des glaces. Le témoignage de Pasqualigo date la découverte, avec cette circonstance spéciale, de l'année 1500. Quant aux cartes Kunstmann III, Kunstmann II et Add. MS 31326, qui expriment la même circonstance dans leur toponymie, elles donnent toutes trois au Groenland le nom de Terre du Labrador, comme le font aussi la plupart des autres cartes de ce temps, inspirées par les Portugais. Mais une carte de type Ribeiro, appelée Wolfenbüttel et datée approximativement de 1527, est plus explicite. Elle porte l'inscription suivante sur le Groenland:

Terre du Labrador, laquelle fut découverte par les Anglais de la ville de Bristol; et, parce que celui qui en donna avis était un labrador des îles Açores, ce nom lui est resté ${ }^{11}$.

On sent ici l'influence de Diogo Ribeiro, qui n'accorde pas cette découverte aux Portugais, mais aux Anglais, tout en con6.

8 Cortesão-Teixeira, Portugaliæ Monumenta cartographica, I: estampa

9 Hoffman, Cabot to Cartier, fig. 9, 55.

10 Ibid., 52.

11 "Tiera del Laborador, la qual fue descubierta por los Ingleses de la vila de Bristol et por $\mathrm{q}$ [ue] dio ell aviso della era labrador de las islas de los açores le quido este nõbre" (Cortesão-Teixeira, Portugaliæ Monumenta cartographica, estampa 12). 
cédant qu'un Portugais des Açores a été le premier à avoir connaissance de cette terre. Ainsi, la date de 1500, cette circonstance d'une découverte inachevée et le nom du labrador qui le premier a vu le Groenland sont reliés ensemble et ne peuvent être disjoints.

Cet argument dispose de quelques spéculations récentes qui voudraient attribuer au labrador une découverte du Groenland en $1496{ }^{12}$, afin de justifier une affirmation d'Alonzo de Santa-Cruz, vers 1541:

(Le Groenland) fut appelé terre du Labrador parce que ce fut un labrador des îles des Açores qui en donna avis et information au Roi d'Angleterre, quand celui-ci l'envoya découvrir par Antonio Gaboto, pilote anglais et père de Sébastien Gaboto qui fut pilote majeur de Votre Majesté ... ${ }^{13}$.

Santa-Cruz, dont le texte mêle constamment la légende aux faits, est une autorité qu'il ne faut accepter qu'avec critique et qu'on ne doit pas presser de trop près. La première partie de ce témoignage répète ce qu'on sait déjà par la carte Wolfenbüttel. Pour la seconde, Santa-Cruz a cité sa source, qui est la Schondia de Ziegler, publiée en $1532^{14}$. Mais la Schondia elle-

12 Theodore Layng, "Charting the Course to Canada", dans Actas do Congresso internacional de Historia dos Descobrimentos (Lisbõa, 1961), II : 255-276.

13 "Fué dicha tierra del Labrador porque dio della aviso e indicio un labrador de las islas de los Açores al Rey de Inglatierra quando el la enbio a descubrir por Antonio Gaboto, piloto ingles y padre de Sebastian Gaboto, piloto mayor que fué de Vuestra Magestad. . " (Biggar, Les Précurseurs, 184).

14 Jacob Ziegler, Schondia (Argentoracti, 1532), f. XCII verso. L'ouvrage de Ziegler étant très rare, nous citons ce qui se rapporte à Sébastien Cabot: "Petrus Martyr Mediolanensis in hispanicis nauigatiõibus scribit, Antonium quendam Cabotum soluentem à Britannia, nauigasse continue uersus septentrionem, quoad incideret in crustas glatiales mense Iulio, inde ergo conuersum remigasse continue secundum littus sese incuruans austrum uersus, donec ueniret ad situm contra Hispaniam supra Cubam insulam Canibalum. Quæ narratio nõ nihil causæ dedit mihi ut Gronlandiam ultra Huitsarch Vuardhus castrum, \& id feci eò libentius q[uod] et senior Archiepiscopus Nidrosiensis constanter affirmabat, mare illic in anconem curuari... Fecit et hoc ad coniecturam $q$ [uod] Cabotus fertur in glatiem incidisse. Et quamuis de mense Iulio contendam non bene relatum esse, adeo si sub polo quoque ipso nauigatum foret: ob eà quæ in eam rem antea disseruimus: tamen $\mathrm{q}$ [uod] aliquo tempore per glatiem nauigauit, id testatur $\mathrm{q}[$ uod] non per mare uastum, sed propinquis littoribus in sinus 
même reproduit, à propos des Cabot, le récit que Sébastien a fait à Pierre-Martyr d'Anghiera d'une découverte qu'il s'attribuait en propre ${ }^{15}$. Le récit de Sébastien est manifestement faux. Ziegler a changé en Antoninus le nom de Sébastien, qui se trouvait dans Pierre-Martyr. Santa-Cruz a modifié Antoninus en Antonio, qu'il a fait père de Sébastien. Sébastien est devenu son propre père. Il est évident que ce témoignage ne contrebalance pas ceux que nous avons donnés plus haut. On ne saurait privilégier un témoignage dont Sébastien Cabot est la source.

La tradition cartographique et cosmographique portugaise semble n'avoir voulu se souvenir que de cette découverte du Groenland qui avait été faite par un labrador en 1500, les Anglais n'employant pas le mot Labrador et lui préférant le terme New found Island ou New found Land. Mais Diogo Ribeiro, un excellent cartographe portugais qui donna une belle impulsion à la cartographie espagnole et qui avait peut-être quelque grief contre son pays d'origine, est peu sympathique aux revendications portugaises. Il inscrit cette remarque sur l'un de ses dessins du Groenland, en 1529 :

Terre du Labrador, laquelle les Anglais de la ville de Bristol découvrirent et dans laquelle ils ne trouvèrent aucune chose profitable ${ }^{16}$.

Il serait temps de passer maintenant aux sources anglaises. Mais voyons d'abord qui était ce labrador des îles Açores. Ernesto do Canto l'a identifié en $1892^{17}$ avec un certain Jean Fernandes, qu'un document de 1506 appelle llavrador ${ }^{18}$ et dont

formam comprenhensum nauigarit, quando ob eandem caussam sinus Gothanus cõcrescat, quoniam strictus est, \& fluuiorum plurium \& magnorum ostia salsam naturam in parua copia superant". L'ouvrage original se trouve à la John Carter Brown Library, Povidence, R.I.

15 Pierre-Martyr d'Anghiera, De Orbe novo decades (Alcala, 1516), III, lib. VI: 52.

16 "Tierra del Labrador laqual descubriero los Ingleses da villa de Bristol en la qual no allaro cosa de ne (?) provecho" (Carte Diogo Ribeiro, Planisphère de 1529, Vatican, dans Cortesâo, Portugaliæ Monumenta cartographica, I: estampa 39).

17 Ernesto do Canto, "João Fernandes, Labrador", Archivo dos Açores, XII (1892).

18 Biggar, Les Précurseurs, 98. 
il est assuré qu'il a fait des découvertes dans le nord de l'Atlantique ${ }^{19}$. Comme cette identité n'a pas été contestée et ne paraît pas contestable, il n'y a pas lieu de la discuter davantage.

On possède encore les lettres patentes que le roi Manuel octroya à Fernandes, le 28 octobre 1499, lui permettant de découvrir des îles nouvelles à ses propres frais et lui promettant la capitanie des terres découvertes par lui avec les privilèges ordinairement attachés à cette charge dans les autres possessions portugaises ${ }^{20}$. Il est certain qu'il a fait de telles découvertes, parce que le fils d'un compatriote et compagnon de son expédition sera doté d'un privilège royal, en 1509 , en "considération des services que nous avons reçus de Pero de Barcelos, déjà mort, qui fut résident de notre île Terceira, dans l'armement et la découverte de la partie du nord" 21. Pierre de Barcelos a déclaré lui-même, en 1506, qu'il était allé à la découverte, "moi et un certain Jean Fernandes, llavrador, à laquelle découverte nous avons été trois bonnes années" ${ }^{22}$.

Durant l'hiver de 1500-1501, le labrador Jean Fernandes, revenu de sa course au nord, se trouvait à Bristol, Angleterre, où il intéressa trois marchands de cette ville à une nouvelle expédition. Le 19 mars 1501, lui-même et deux autres Açoréens, ses associés François Fernandes et Jean Gonzales, en compagnie de trois marchands de Bristol, Richard Warde, Thomas Ashurst et John Thomas, obtenaient une charte de Henri VII pour entreprendre cette expédition ${ }^{23}$. C'est de la sorte que le labrador a donné avis de sa découverte au roi d'Angleterre. Il est assez probable que Fernandes avait envoyé un messager au Portugal pour donner un rapport de son premier voyage, car dans ce pays, on était déjà en 1501 informé de la découverte de l'année précédente.

\section{Ibid., 100-101.}

20 Ibid., 31.

21 “... avemdo nos respeito aos serviços que temos recebidos de Pero de Barcelos, ja finado, morador que foy [na] nosa ilha Terçeira, narmaçam e descubrimento da parte de norte..." (Biggar, Les Précurseurs, 100-101). 22 “... eu e hum Johã Fernandes, llavrador, no quall descobrimento andamos bons tres anos..." (Biggar, Les Précurseurs, 98).

23 Biggar, Les Précurseurs, 40-50. 
Il paraît assuré que l'association anglo-portugaise de Bristol a fait une expédition au Groenland. Une vieille chronique de la ville de Londres mentionnait, à la $\mathrm{XVII}^{\mathrm{e}}$ année du règne de Henri VII:

This yere three men were brought out of an Iland founde by merchauntes of Bristow forre beyonde Ireland, the which were clothed in Beestes skynnes and ete raw fflessch, and rude in their demaenure as Beestes ${ }^{24}$.

La description serait générale à l'excès, si elle ne contenait ce trait caractéristique, que ces indigènes étaient des mangeurs de viande crue. Cette coutume n'est pas celle des Indiens d'Amérique, mais bien des Esquimaux. Ce détail, rapproché de ce qu'on sait de l'association entre Jean Fernandes et les marchands de Bristol et confirmé par le témoignage plus haut cité de Ribeiro, ne laisse pas de doute sur la réalité d'une expédition réussie au Groenland, en 1501 ou en 1502.

La difficulté est de savoir quelle des deux années a été celle du voyage, qui pouvait se faire seulement en été. La dix-septième année du règne de Henri VII commençait le 22 août 1501 et finissait le 21 août $1502^{25}$. Les préparatifs du voyage, ou d'autres raisons, ont pu retarder l'expédition jusqu'à 1502. Car en 1506, Pierre de Barcelos, qui avait accompagné Jean Fernandes, donnait trois bonnes années comme longueur à leur absence des Açores ${ }^{26}$. Fernandes, ayant quitté sa patrie à la fin de 1499 ou au début de 1500, n'y serait revenu qu'à la fin de 1502 ou au début de 1503 . Un retour aussi tardif supposerait que l'exploration du Groenland a eu lieu en 1502 plutôt qu'en 1501. En tout cas, de nouvelles patentes de Henri VII à Jean Gonzales, François Fernandes, Hugh Eliot et Thomas Ashurst, du 9 décembre 1502, ne mentionnent plus le

24 Biggar, Les Précurseurs, 100.

25 Le règne de Henri VII a commencé le 22 août 1485 , date de la victoire de Bosworth. Mais, selon Biggar (Les Précurseurs, 100 n. 1), les années du Chronicon dont ce renseignement est tiré auraient couru du $\mathbf{1 5}$ septembre au 14 septembre.

26 Voir plus haut, note 20. Nous corrigeons ici la date de 1503, que nous avions donnée à ce voyage ("Jean Cabot et la découverte de l'Amérique", Revue d'Histoire de l'Am. Fr., XIX (1965) : 400-401 n. 29). 
nom du labrador, qui a sans doute décidé de ne pas participer à un autre voyage ${ }^{27}$.

Que cette découverte du Groenland, en 1501 ou en 1502, soit la plus ancienne que les Anglais aient pu faire dans le nord de l'Atlantique, les vers de John Rastell, écrits en $1519{ }^{28}$, le démontrent clairement:

This See is called the great Occyan.

So great it is that never man

Coude tell it sith the worlde began,

Tyll now within this XX. yere

Westwarde be founde new landes

That we never harde tell of before this

By wrytynge nor other meanys.

Les vingt dernières années de Rastell ne peuvent commencer qu'en 1499. Le poète a lu les voyages d'Améric Vespuce, publiés par Waldseemüller en $1507^{29}$ et le premier de ces voyages est justement daté de 1499 .

But this Newe Landes founde lately

Ben callyd America bycause only

Americus dyd furst them fynde.

Autant que sache l'auteur de ces vers, le roi d'Angleterre fut le premier souverain à envoyer une expédition officielle dans ce nouveau monde:

There into so farre a grounde

Whiche the noble kinge of late memory,

The moste wyse prynce the VII Henry,

Causyd furst for to be founde.

Comme cette commission royale doit nécessairement prendre place "within this XX. yere", il ne peut s'agir ici que de l'expédition anglo-portugaise de 1501 ou de 1502. L'écrivain regrette amèrement que les Anglais n'aient pas pris leur part

27 Biggar, Les Précurseurs, 70-81.

28 James A. Williamson, The Voyages of the Cabots and the English Discovery of North America under Henry VII and Henry VIII (London, 1929), 84-92.

29 Martin Waldseemuller, Cosmographiæ introductio... (Saint-Dié, 1507), sans pagination. 
du nouveau continent. Il fait allusion à un voyage qu'il a luimême tenté en $1517^{30}$, ainsi qu'à quelques autres tentatives anglaises avortées:

But yet not longe ago

Some men of this countrey went

By the Kynges noble consent

It for to serche to that entent

And coude not be brought therto,

But they that were they venteres

Have cause to curse their maryners,

Fals of promys and dissemblers,

That falsly them betrayed,

Whiche wolde take no paine to saile farther

Than their owne lyst and pleasure.

Wherfore that vyage and dyvers other

Suche kaytiffes have distroyed.

Mais durant le même temps, les marins d'autres nations, déjà en 1519, ont parcouru les côtes du nouveau-monde et ils rapportent ses dépouilles dans leurs pays respectifs:

Yet many now have ben there

And that countrey is so large of rome,

Muche lenger than all Cristendom,

Without fable or gyle.

For dyvers maryners have it tryed,

And sayled streyght by the coste syde

Above V thousand myle.

Nowe Frenchemen and other founde the trade,

That yerely of fysche there they lade

Above an C sayle.

Remarquons en terminant que John Rastell tient pour un seul continent, qu'il allonge sur une distance de cinq mille milles, toutes les découvertes encore éparses qui ont été faites dans le nouveau-monde. Déjà cette opinion apparaissait dans la lettre de Pietro Pasqualigo à la Seigneurie de Venise, le 18 août $1501^{31}$. On peut dire qu'elle a été générale, dès les premières années du seizième siècle. D'autant plus qu'on croyait

30 Voir la documentation dans Williamson, op. cit., $85 \mathrm{ss}$.

31 Biggar, Les Précurseurs, 65-66. 
au début avoir touché le littoral de l'Asie. A cela ne s'oppose pas le fait que plusieurs cartographes, par un louable souci d'objectivité, isolaient les différentes découvertes dans la mer océane. Mais la tendance à les relier s'est bientôt fait sentir. Ruysch, dès 1507, réunit le Groenland à Terre-Neuve. En 1530, toute la côte nord-américaine apparaîtra comme un littoral continu, alors qu'une importante section n'en aura pas encore été explorée.

\section{DATE DE LA DECOUVERTE DE TERRE-NEUVE}

Nous avons vu plus haut comment la redécouverte du Groenland par Jean Fernandes, en 1500, ouvre l'ère des explorations réussies dans le nord-ouest de l'Atlantique. Mais il ne nous semble pas historiquement prouvé que Gaspar Corte-Real, soit en compagnie de Fernandes, soit pour son propre compte ${ }^{32}$, ait passé en vue du Groenland, cette même année, avant de toucher les côtes de notre Labrador et de Terre-Neuve. On sait que Gaspar Corte-Real a reçu, le 12 mai 1500 , six mois et demi après Fernandes, des lettres patentes l'autorisant à poursuivre les efforts déjà commencés à ses propres frais pour découvrir de nouvelles terres ${ }^{33}$. Ce document et ceux qui rapporteront plus tard les succès et les malheurs des Corte-Real laissent bien entendre qu'à la date du 12 mai 1500 , on ne connaissait encore rien au Portugal de l'existence d'îles ou de continent dans cette partie de l'océan ${ }^{34}$. Les découvertes certaines du Portugal en

32 Cette alternative est exposée par Biggar (Les Précurseurs, xiv-xv). 33 Texte portugais dans Biggar, Les Précurseurs, 32-35; aussi dans Harrisse, Les Corte-Real ...., 196-199.

34 Quelques historiens portugais auraient tendance à faire de Jean Vaz Corte-Real, père de Gaspar et de Miguel, le premier découvreur de la côte américaine, en 1471-1472. Le fondement historique le plus valable est la mention de terres et d'îles au nom de ce gentilhomme dans le nord sur des cartes de 1534,1569 et 1571 . Mais les inscriptions cartographiques, surtout à cette époque tardive, ont une trop faible valeur, en l'absence d'autres preuves (v. E. Brazâo, The Corte-Real family and the new World (Lisbon, 1965), 32-47). Il paraît plus sûr de s'appuyer sur des affirmations explicites comme celle-ci, dans les lettres patentes du roi Manuel à Vasco Anes Corte-Real, le 17 septembre 1506: "... como ho dicto Gaspar Corte-Reall, seu irmãao, foy o primeiro descubridor das dictas terras a sua propria custa \& despessa..." (Biggar, Les Précurseurs, 93). 
Amérique du Nord sont postérieures à cette date ${ }^{35}$. En cette matière, en effet, le silence est significatif, vu l'émoi que provoque toujours l'annonce d'une découverte. Si stricte que soit, à l'égard des étrangers, la politique du silence et du secret, il est impossible à un roi de n'en pas conférer avec ses sujets et il en transpire toujours quelque chose.

Autant les sources sont affirmatives et dignes de confiance à propos du fait central, la découverte d'une partie de la côte nord-américaine par Gaspar Corte-Real, autant elles fourmillent de désaccords et de contradictions sur les circonstances de ce fait. De toute nécessité, il faut choisir le témoignage le plus autorisé, lorsqu'il s'agit de rétablir les circonstances véritables. Dans notre cas, la "ratification" donnée par le roi Manuel à Miguel Corte-Real des donations que son frère lui a faites est sans conteste la plus autorisée de nos sources ${ }^{36}$. On y trouve en effet la version des deux témoins les mieux informés de l'événement: le roi, qui a donné commission au découvreur, et le frère de celui-ci qui est en même temps son associé.

Ce document servira principalement à dirimer les problèmes que posent le nombre des voyages faits par les Corte-Real, les dates de ces voyages et la participation de Miguel à ces expéditions. En réalité, tous ces problèmes n'en font qu'un, puisque la question peut se résumer à savoir si Miguel CorteReal a accompagné son frère à Terre-Neuve en 1501. On sait en effet que Miguel a pris part à la seconde expédition, alors que Gaspar avait été seul à diriger la première: "pour autant

35 Nous ne nions pas que les Portugais aient fait beaucoup d'efforts, même dans le nord de l'Atlantique, pour découvrir des terres nouvelles, et cela même avant Fernandes et Corte-Real. Les souverains portugais avaient coutume de promettre à ces aventuriers, qui risquaient souvent tout ce qu'ils avaient dans ces entreprises, le gouvernement et la jouissance des terres qu'ils découvriraient. Les lettres patentes de Fernandes et de CorteReal ont le style ordinaire de ces documents. Celles qui furent accordées au second sont toutefois fort prolixes, à cause sans doute du noble lignage de la famille Corte-Real. Mais de réussites de telles entreprises, les premières qui soient historiquement attestées, en ce qui concerne le nord, autant que nous sachions, sont celles de Fernandes et de Corte-Real.

36 Texte portugais dans Biggar, Les Précurseurs, 67-69; aussi dans Harrisse, Les Corte-Real ..., 214-215. 
que ledit Miguell Corte-Reall avait fait beaucoup de frais et de dépenses de sa fortune dans ladite découverte, aussi bien dans lesdits navires que ledit sien frère a armés pour cela la première fois qu'il a trouvé ladite terre, que cette seconde [fois] où maintenant il a été au même titre que lui ... ${ }^{37} "$. Si Miguel est allé à Terre-Neuve en 1501, on pourra attribuer à Gaspar une expédition faite en 1500, où il aurait découvert le pays nouveau. Et ainsi, le voyage que Miguel prépare au début de 1502 pour aller à la recherche de Gaspar devrait être compté comme le troisième des Corte-Real, parce qu'il est assuré qu'il y a eu un voyage en 1501. Mais si Miguel n'est pas allé à Terre-Neuve en 1501, le deuxième voyage, auquel il prend une part de premier plan, est celui de 1502 et le premier, fait par Gaspar seul, est celui de 1501. Cette dernière année, de la sorte, devient celle de la découverte de Terre-Neuve.

Déjà, les lettres d'Albert Cantino, du 17 octobre $1501^{38}$, et de Pietro Pasqualigo, du 18 et du 19 octobre $1501^{39}$, datent clairement la découverte de l'année 1501. La difficulté vient de l'obscurité des termes de la "ratification", qui demeure notre témoignage le plus autorisé. Il nous faut faire appel à d'autres témoignages incontestables, pour y jeter de la lumière.

On constate la présence de Gaspar Corte-Real au Portugal le 12 mai 1500, jour où il a obtenu les lettres patentes déjà mentionnées. Et on l'y retrouve encore le 21 avril 1501, jour où il signe à Lisbonne un reçu pour des vivres destinés à un prochain voyage ${ }^{40}$. S'il est allé à Terre-Neuve en 1500 , il n'a pu partir qu'après le 12 mai et il est revenu passer l'hiver au Portugal.

37 "por quamto elle dito Miguell Corte Reall tinha feito muyto gasto e despesa de sua fazemda no dito descobrimento, asy nos ditos navyos que ho dito seu irmaão pera ella armou per a primeira vez que a dita terra achou, e asy desta segumda que ora foy como com elle" (Biggar, Les Précurseurs, 68).

38 Texte italien dans Biggar, Les Précurseurs, 61-63; au complet dans Harrisse, Les Corte-Real..., 204-208.

39 Texte italien, dans Biggar, Les Précurseurs, 65-66; aussi dans Harrisse, Les Corte-Real..., 209-211. La lettre du 19 octobre ne se trouve que dans Harrisse, op. cit., 211-212.

40 Texte portugais dans Biggar, Les Précurseurs, 60; Harrisse, Les Corte-Real... Post-scriptum, 6-7. 
Le départ de 1501 n'a pu avoir lieu qu'après le 21 avril. Ces premières dates certaines étant posées, discutons le cas de Miguel. On peut montrer que ce dernier n'a pas accompagné Gaspar en 1501. Certaines sources qu'on invoque dans ce sens établissent plutôt le contraire. Le 4 novembre 1501, le roi Manuel lui accordait une pension annuelle de 30000 reis pour ser-

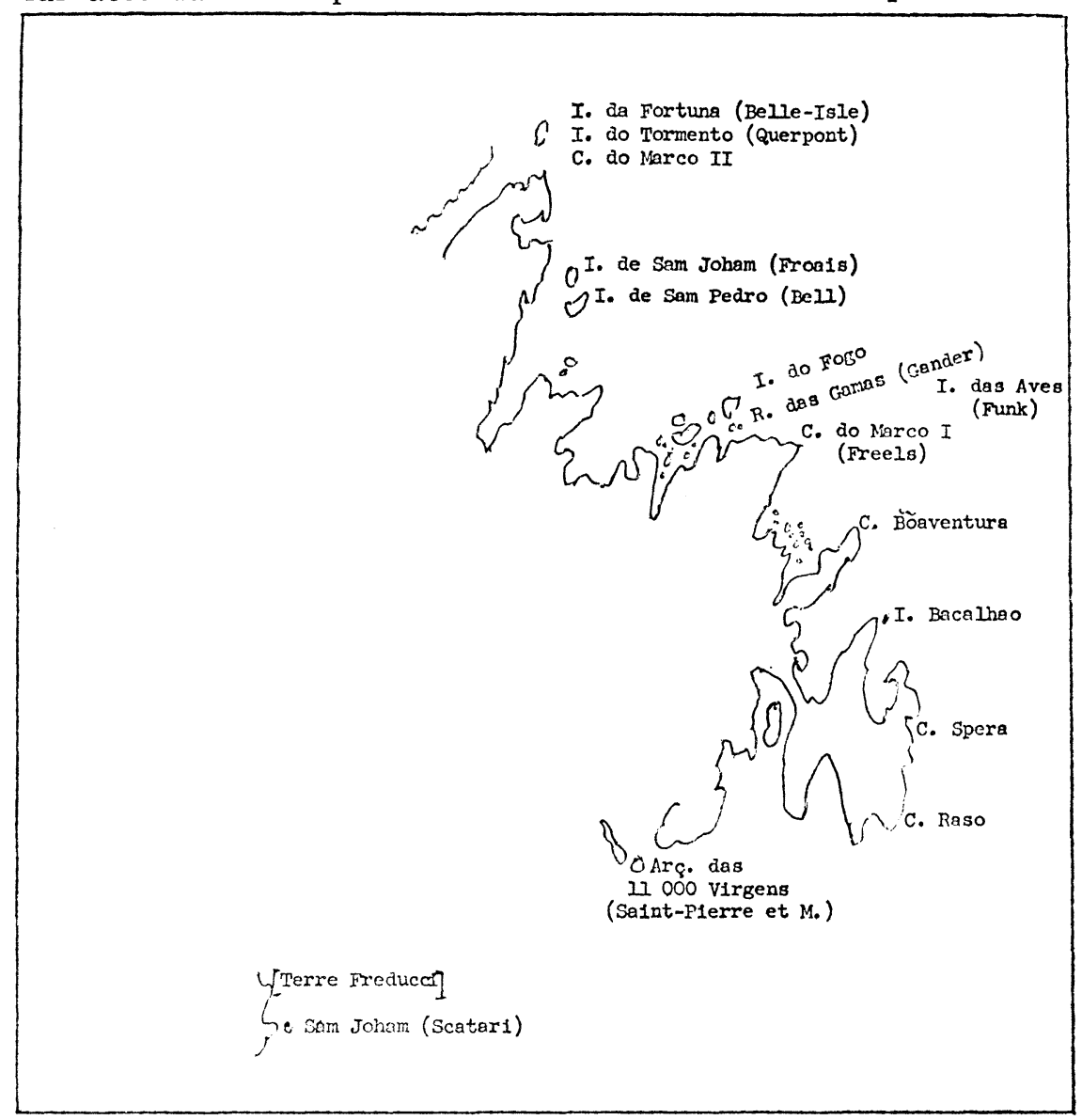

En dépit des cartes produites avant 1534 , ce dessin représente tout ce que la cartographie avait reçu d'information objective sur la côte canadienne avant les explorations de Jacques Cartier. C'est ce que nous appelons la carte portugaise, à laquelle les explorations de Verrazano et de Gomez n'ont rien ajouté d'authentique. 
vices rendus à lui-même et à son prédécesseur Jean II ${ }^{41}$. Parmi ces services nulle mention de découverte. Et d'ailleurs, si la pension avait été donnée à cause de la découverte de TerreNeuve, Gaspar l'aurait méritée le premier. On sait, au surplus, que Miguel, en ce même été 1501 , a pris part à une expédition navale qui n'a rien à voir avec l'exploration de son frère.

On connaît en effet deux notes signées par lui, les 6 et 7 août $1501^{42}$. Elles nous montrent Miguel commandant un navire monté par quatre-vingts hommes. Moins de trois mois auparavant, le gentilhomme avait reçu du roi l'ordre d'embarquer cinquante hommes dans un vaisseau et un nommé Christophe Lopez lui avait fourni des vivres pour entretenir cette troupe durant trois mois. Avant le départ de Lisbonne, un second ordre royal avait obligé Miguel à ajouter trente hommes aux cinquante qu'il avait déjà. On ne put toutefois embarquer aussi des victuailles pour cette recrue additionnelle, faute de place dans le navire. Ayant donc eu à nourrir quatre-vingts hommes avec les provisions de cinquante, le commandant se trouva en

${ }^{41}$ Henry Harrisse, Les Corte-Real..., 213; aussi cité par E. Brazão, The Corte-Real family and the new World, 152-153.

42 A cause de l'importance de ces deux textes pour notre sujet, il nous semble nécessaire de les reproduire ici tels qu'ils l'ont été déjà par E. Brazão, The Corte-Real family and the new World, $165 ; \mathrm{n}$. 6 du même, "Les Corte-Real et le Nouveau-Monde", Revue d'Hist. de l'Am. fr., XIX (1965) : 348, n. 5; voir aussi Harrisse, Les Corte-Real..., 201-202.

1) 6 août 1501

"senhor xpãm lopez quando armey em lyxboa eu tomei mãtimento pera tres meses. S. pera cinquventa homes e depois mandou el rey noso senhor que tomase mais trimta homes pera os quaes nõ pude tomar mais mãtimento por nõ caber no nauyo. aguora ha acerqua de tres (dias) que se gastou de guisa que aribei aqui por migoa dele e por ponẽte que venta que me nõ deyxa ir peçovos por merce que me mãdes dar duas pipas de vinho e hũ boy ou $\mathrm{xv}$ ou $\mathrm{xx}$ aroubas de carne e isto vos peço da parte del rey et peço merce a ffernã dalcaçova que ve a nissycidade minha que vos de disto huma certidã. fecto aos seis dias dagosto de quinhentos e hum".

2) 7 août 1501

" $\mathrm{Eu}$ miguell corte reall diguo $\mathrm{q}$ he verdale (sic) $\mathrm{q}$ reçeby de xpãm lopez escudeiro del rej nosso (senhor) duas pipas de vinho $\mathrm{e} x \mathrm{x}$ arobas de carne as quaes duas pipas de vynho e vynte arobas de carne asy recebo para mãtimento de oytenta homes, aos quæs faleceo ho mãtimento q pera eles trazia aos sete dias do mes dagosto et porq assy he verdade $q$ o dito mãtimento dele recebeo lhe dey este fecto aos sete dias do dito mes dagosto da era de quinhentos e hum - em malega. 
détresse avant d'avoir pu revenir à Lisbonne, à la fois par manque de vivres et à cause d'un vent d'ouest qui arrêtait sa marche. Tel fut le motif des deux billets du 6 et du 7 août, pour demander un nouvel approvisionnement. Le second de ces billets, un reçu pour les vivres embarqués au départ, indique le lieu où se trouve alors le capitaine en détresse, un port appelé Malega.

Une simple réflexion sur le contenu de ces deux notes suffit à convaincre que cette expédition de Miguel ne peut avoir aucun rapport avec la découverte du nouveau-monde. Remarquons d'abord que la relation établie entre les deux par quelques historiens est purement hypothétique. Il n'y en a aucune d'explicitée dans les textes. Et à quoi pouvaient servir cinquante hommes dans un voyage d'exploration, dans un petit navire où quinze ou vingt suffisaient d'ordinaire à la manœuvre ? Et à quoi aurait-on pensé de les munir de vivres pour trois mois seulement ? Un voyage de découverte doit être prêt à affronter l'imprévisible pour le double et même le triple de ce temps. Mais il fût devenu absolument insensé d'embarquer trente nouveaux hommes sans augmenter les vivres. Car les provisions de trois mois pour cinquante hommes ne dureront pas deux mois pour quatre-vingts. On a supposé que Miguel avait l'intention de fonder une colonie. L'entreprise serait devenue plus folle encore en cette hypothèse. Car une colonisation demande un affrètement plus considérable encore qu'un simple voyage. Il est beaucoup plus raisonnable de supposer que Miguel est parti en expédition de guerre contre les Maures ou les Turcs, sur la Méditerranée où il ne devait jamais être bien loin d'un refuge en cas de disette.

Cette hypothèse raisonnable se trouve confirmée, lorsqu'on s'arrête au nom du port où s'est arrêté Miguel Corte-Real: Malega. Compte tenu du peu de cas que l'on fait alors de l'orthographe, on n'hésiterait pas à reconnaître ici Malaga, port de l'Andalousie sur la Méditerranée. Ceux qui font de l'expédition de Miguel un voyage au nouveau-monde ne peuvent consentir à cette identification et situeraient volontiers Malega aux Aço- 
res. Mais on ne connaît aucun lieu de ce nom dans les îles. D'après le billet, Malega est un port d'où l'on peut facilement communiquer avec Lisbonne, où résidait sans doute Christophe Lopez ${ }^{43}$. Le navigateur s'y est arrêté "à cause du manque de munitions et d'un vent du ponent qui souffle et ne lui permet pas de poursuivre" ${ }^{44}$. Si un vent d'ouest empêche le marin d'avancer, c'est que son navire se dirige vers l'ouest, contre le vent. Mais, lorsqu'on vient du nouveau-monde, un vent d'ouest, loin d'être un obstacle, est ce qu'on peut désirer de mieux. Miguel a donc été envoyé en Méditerranée durant l'été de 1501 et l'on n'a pas de raisons sérieuses de douter que Malega soit réellement Malaga. Ainsi, il n'a pu accompagner Gaspar, son frère, durant la même période. Car s'il avait à ce moment rapporté des nouvelles de ce dernier, il lui eût été difficile de n'en rien communiquer par le courrier qui portait ses lettres au Portugal. Les nouvelles de Terre-Neuve, autant qu'on sache, ne sont arrivées qu'au mois d'octobre.

Enfin, Harrisse, renversant résolument une opinion à laquelle il venait d'adhérer, a lui-même fourni la preuve définitive que Miguel n'avait pas pris part au voyage de découverte de 1501. Peu après la publication de son livre, Les Corte-Real et leurs voyages au Nouveau-Monde, en 1883, il reçut de M. de Brito Rebello une copie d'un nouveau document, un second reçu de Miguel à Christophe Lopez pour une douzaine de merlans, daté aussi de Malega le 7 août 1501. Laissons parler l'infatigable historien des découvertes: "Or au verso de ce reçu se trouvent d'autres quittances du même genre, données par deux capitaines, dont les caravelles suivaient ou précédaient celle que montait Miguel Corte-Real, et faisant toutes parties de l'escadre envoyée par le roi Manuel, sur les instances du pape Alexandre VI, contre le Grand Turc (Bajazet II) pour sauvegarder les possessions grecques de la République de Venise. Cette expé-

43 Lopez est loin du lieu où se trouve Miguel. Il est requis d'envoyer une reconnaissance à Fernand d'Alcaçova, qui à ce moment fournit Miguel de vivres. Comme Lopez était à Lisbonne au départ du navire, il s'y trouve vraisemblablement encore. D'autant plus que le reçu de Miguel est destiné à être présenté aux officiers royaux chargés de défrayer Lopez. 44 "por migoa dele e por ponẽte que venta que me nõ deyxa ir". 
dition, composée de trente voiles, portant 3500 hommes, et commandée par João de Menezes, appareilla de Lisbonne le 15 juin 1501. Les Turcs s'étant désistés de leurs projets d'attaque contre les îles de l'Archipel, le gros de la flotte portugaise, qui avait été précédé en novembre par plusieurs caravelles au nombre desquelles se trouvait celle que montait Miguel CorteReal, revint à Lisbonne au mois de décembre suivant ${ }^{45}$.

Par conséquent, le premier voyage de Gaspar Corte-Real, celui où il découvrit la côte nord-américaine, a eu lieu en 1501 et la seconde expédition, à laquelle Miguel a participé au même titre que son frère, est celle de 1502.

C'est également ce qui ressort d'une analyse un peu serrée du texte de la "ratification". En voici une première tranche essentielle: "Il (Miguel) nous a dit maintenant (ora) que, voyant comment Gaspar Corte-Real, son frère, était parti, il y a nombre de jours, avec trois navires pour découvrir une terre nouvelle, de laquelle il avait déjà (au moment où Miguel parlait, non à celui où Gaspar était parti) découvert une partie, comment ensuite, après un certain temps, deux desdits navires étaient revenus à ladite cité - ils avaient [été] cinq mois ${ }^{46}$ et que lui-même n'était pas revenu, il (Miguel) voulait aller le chercher" ${ }^{47}$. La circonstance qu'il faut retenir est celle des trois navires, avec lesquels Gaspar Corte-Real est parti et dont l'un, celui du découvreur, n'est pas revenu. Le texte enchaîne ensuite: "et que pour autant que ledit Miguel Corte-Real avait fait beaucoup de frais et de dépenses de ses biens dans ladite découverte, aussi bien dans lesdits navires (c'est-à-dire les trois qui

45 Harrisse, Les Corte-Real..., Post-Scriptum, 15-16.

46 C'est ainsi que nous pensons devoir traduire "averiam cinquo messes". Il n'y avait pas encore cinq mois depuis octobre, où l'on voit arriver un navire. Et l'autre a dû arriver encore plus tard, puisque ni Cantino ni Pasqualigo n'en avaient eu connaissance encore au 18 octobre. D'autre part, de mai à octobre, durée probable du voyage, il y a cinq mois.

47 “... nos disse ora, que vemdo elle como Gaspar Corte Reall, seu irmaao, avia dias que partira desta cidade com tres navyos a descobrir terra nova, de quall ja tinha achada parte della, e como depois de passado tempo vieram dous dos ditos navyos aa dita cidade, averiam cinquo messes, e elle nam vinha, que elle a queria hyr buscar" (Biggar, Les Précurseurs, $67-68)$. 
sont partis avec Gaspar et dont l'un n'est pas revenu) que ledit sien frère a armés pour cela la première fois qu'il a trouvé ladite terre, que cette seconde (fois) où maintenant il a été (foy) au même titre que lui (como com elle)" ${ }^{48}$. Le texte laisse bien entendre que l'expédition où la terre nouvelle a été découverte et celle dont Gaspar Corte-Real n'est pas revenu au Portugal n'en est qu'une seule. Quant au verbe foy, qui désigne la participation de Miguel à la seconde expédition, il ne signifie pas que ce dernier est allé au nouveau-monde, mais qu'il fut (foy) dans l'armement de ce nouveau voyage tout comme son frère, alors que Gaspar seul avait fait l'armement du précédent, auquel Miguel n'avait contribué que de son argent.

Pour Miguel Corte-Real, Gaspar est parti et il n'est plus revenu après sa découverte. Et cela est corroboré par les témoignages de Cantino et de Pasqualigo, bien que ceux-ci ne comptent que deux navires dans l'expédition. Voici ce qu'en dit le premier: "L'autre (navire) compagnon a décidé de poursuivre le long de cette côte aussi loin qu'il faudra pour reconnaître si elle est une île ou bien une terre ferme. Et ainsi, le Roi attend avec grand désir et celui-là et d'autres aussi, desquels, lorsqu'ils seront venus et auront apporté des nouvelles dignes de Votre Excellence, je lui donnerai aussitôt avis..." ${ }_{49}$ Et Pasqualigo: "On attend de jour en jour l'autre caravelle capitanesse, de laquelle on obtiendra des précisions sur la qualité et les conditions de la susdite terre, parce qu'elle est allée plus avant, parcourant cette côte pour en découvrir le plus possible..." " 50 De même à ses frères: “... et avec l'autre caravelle

48 "e que por quamto elle dito Miguell Corte Reall tinha feito muyto gasto e despesa de sua fazemda no dito descobrimento, asy nos ditos navyos que ho dito seu irmaão pera ella armou per a primeira vez que a dita terra achou, e asy desta segumda que ora foy como com elle" (Biggar, Les Précurseurs, 68).

49 "L'altro compagno ha deliberato andar tanto per quella costa che vole intendere se quella è insula o pur terra ferma. Et cusi il Re con molto desiderio et quello et altri aspecta, liquali venuti che siano, et portando cosa digna de Vostra Excellentia, subito ne daro notitia a quella" (Biggar, Les Précurseurs, 62).

50 "Expetasse di zorno l'altra caravella capetania, da laqual distinctamente se intendera la qualità et condition ch'è la sopradita terra, per esser andata piu avanti, scorendo per quella costa, per discoprir quanto piu potra di quella" (Biggar, Les Précurseurs, 66). 
qu'on attend d'heure en heure, il en vient cinquante autres (indigènes) ... Et si l'on entend autre chose à la venue de la caravelle capitanesse, je vous en aviserai semblablement" 51. Puis la carte Cantino, dressée par un Portugais contemporain de la découverte, déclare avec toute la clarté désirable: "Cette terre a été découverte par le commandement $d u$ très haut et très excellent prince-roi, dom Manuel, roi de Portugal. Celui qui la découvrit fut Gaspar de Corte-Real, chevalier dans la maison dudit roi, lequel, quand il la découvrit, envoya un navire avec certains hommes et femmes qu'il avait trouvés dans ladite terre, et lui-même demeura avec un autre navire et ne revint jamais. Et l'on croit qu'il s'est perdu." ${ }^{2}$ Enfin, tout en étant moins claire que le texte précédent, la donation à Vasco Anes CorteReal, du 17 septembre 1506, ne parle pas non plus de deux voyages de Gaspar: "ayant considération et souvenir de la manière dont ledit Gaspar Corte-Real, son frère, a été le premier découvreur desdites terres à ses propres frais et dépens, avec beaucoup de travail et de risque de sa propre personne, et dont finalement, avec plusieurs serviteurs et (autres) hommes qu'il avait avec lui, il est mort dans cette entreprise" ${ }^{53}$.

Il y a donc complet accord des témoignages contemporains sur le fait principal: Terre-Neuve a été découverte en 1501, au cours d'une expédition dont le chef ne revint jamais dans sa patrie. Comment la légende d'un voyage de Corte-Real en 1500, au cours duquel il aurait passé au Groenland et découvert TerreNeuve, a-t-elle pu naître ? La réponse ne saurait être catégo-

51 “... et cum l'altra caravella che sa aspecta d'hora in hora ne vien altri cinquanta... Et se altro sa intendera nella venuta de la Caravella capitania similiter ve notificaro" (Harrisse, Les Corte-Real..., 212).

52 "Esta terra he descoberta per mandado do muy alto excelentissimo principe Rey dom manuell Rey de portugall a qual descobrio gaspar de corte real caualleiro na cassa do dito rey, o quall quãdo a descobrio mandou hũ naujo com certos omes \& molheres que achou na dita terra \& elle ficou con outro naujo \& nũca mais veo \& crese que he perdido" (Cortesão \& Teixeira da Mota, Portugaliæ Monumenta Cartographica (Lisbõa, 1960), I: estampas 4 et 5).

53 "avendo respecto \& lembramça como ho dicto Gaspar Corte Reall, seu irmãao, foy o primeiro descubridor das dictas terras a sua propria custa $\&$ despessa, como ( finalmente com muitos criados \& homes, que comsigo levava, nisso acabou" (Biggar, Les Précurseurs, 93). 
rique, mais on peut proposer celle-ci. Le nom de Fernandes a été profondément oublié par les historiens portugais. Et le Groenland a été vu comme un littoral continuant celui de TerreNeuve. Il semble qu'on ait mis tout cet unique continent au compte de Corte-Real et qu'on ait attribué à celui-ci même le voyage de Fernandes en 1500 . Cette vue apparaît un peu après 1520 , sur la carte Kunstmann IV, où l'inscription sur TerreNeuve se lit comme suit: "Gaspar Corte-Regalis, Portugais, est celui qui a le premier trouvé cette terre. Il emmena avec lui des hommes sauvages et des ours blancs. Il s'y trouve une grande abondance d'animaux, d'oiseaux et aussi de poissons. L'année suivante, il (Corte-Real) fit naufrage et ne revint jamais. Ainsi arriva-t-il à son frère Miguel l'année suivante ${ }^{54} "$. Ici, on semble indiquer trois voyages des Corte-Real et si Gaspar et Miguel se sont perdus respectivement en 1501 et en 1502, le premier voyage et la découverte de Gaspar ont nécessairement eu lieu en 1500. Mais cette carte n'a pas été faite au Portugal, comme le montre le latin international qui y est employé; et l'information y est composite. Les ours blancs ne sont tout de même pas communs à Terre-Neuve, où il peuvent cependant arriver, portés par les banquises. Les plus anciens historiens portugais à s'occuper des découvertes nordiques ont adopté d'emblée la version d'un voyage de Gaspar Corte-Real en 1500. Damião de Goes est le principal d'entre eux ${ }^{55}$. D'après lui, Gaspar aurait découvert alors une terre très froide qu'il au-

54 "Terram istam Gaspar Corte Regalis portugalensis primo invenit secum tulit hoẽs silvestres et ursos albos in ea esse magna multitudo animalium et avium necnon et pisciũ qui anno sequenti naufragium perpessus unq rediit sic et fratri eius michaeli anno sequenti contigit" (Cortesão \& Teixeira da Mota, Portugalix Monumenta cartographica, I: estampa 12). En regard de ce témoignage chronologiquement imprécis, on peut cependant mettre celui du globe de Jean Schöner (1520), à propos de la Terra Corte Realis: "Hec terra inventa est ex mandato regis portugalliæ per Capitaniam Gaspar Cortereali: Anno christi 1501" (Kretschmer, Die Entdeckung Amerika's..., Tafel XIII).

55 Damião de Goes, Chronica do felicissimo rey dom Emanuel da gloriosa memoria, a qual por mandado do serenissimo principe, o infante dom Henrique seu filho, o cardẽal de Portugal, do titulo dos Santos quatro corõados, Damião de Goes coligio \& compos... (Lisbõa, 1566-1567). Ce qui se rapporte au voyage de 1500 est cité par Eduardo Brazão, "Les CorteReal et le Nouveau monde", Revue d'Hist. de l'Am. fr., XIX: 180-181; voir aussi Harrisse, Les Corte-Real..., 223-235. 
rait appelée Terre verte. Ce nom n'est que la traduction de Groenland et il n'est pas attesté durant le premier quart du seizième siècle. La description faite par l'historien pourrait se rapporter en partie à Terre-Neuve et en partie au Groenland. Elle est en tout cas assez confuse. Un autre historien, Antonio Galvão, adopte aussi la date de $1500{ }^{56}$. Sa description est plus précise, mais plus brève; et il ne semble pas douteux qu'elle se rapporte à Terre-Neuve. Ces précurseurs vont faire loi dans l'historiographie du nouveau-monde et la légende des trois voyages des Corte-Real et d'une découverte du Groenland par eux va se trouver établie.

Les sources contemporaines varient considérablement sur les détails. Cantino et Pasqualigo ne composent l'expédition de 1501 que de deux navires, dont un seul est revenu à Lisbonne. La "ratification", qui parle de trois navires et du retour de deux d'entre eux, paraît être plus digne de confiance, à cause de la plus grande autorité du roi et de Miguel Corte-Real. Cantino et Pasqualigo, écrivant le premier, le 17 octobre 1501, le second, le 18 et le 19, n'ont vu chacun revenir qu'un seul vaisseau, mais à des dates différentes: le premier, le 11 octobre; le second, le 8 ou le 9 du même mois. Il est plus probable qu'il s'agit du même arrivage, car, au temps où ils écrivent, chacun d'eux n'aurait pu ignorer le retour d'un second navire. Le deuxième vaisseau n'aurait donc pu arriver qu'après le 19 octobre ${ }^{57}$. Ces témoins diffèrent aussi à propos de la date du départ de l'expédition, que Cantino place en février 1501, tandis que Pasqualigo le fait remonter à l'année 1500. Mais comme il est certain que Gaspar Corte-Real se trouvait encore à Lisbonne le 21 avril $1501{ }^{58}$, le départ n'a pu avoir lieu qu'après cette date. Il serait oiseux de supposer des départs séparés des navires, à des dates

${ }^{56}$ Antonio Galvão, Tratado dos descobrimentos antigos, e modernos feitos até a era de 1550. com os nomes particulares das pessõas que os fizerão... (Lisboa, 1563). Citation par Brazão, art. cit., ibid., 181-182, v. Harrisse, Les Corte-Real..., 223.

57 La conciliation des témoignages paraît impossible. Miguel CorteReal dit bien que deux navires sont revenus de Terre-Neuve. Et la carte Cantino maintient qu'un seul retourna à Lisbonne.

58 Voir le texte portugais du reçu qu'il signe à Lisbonne, à cette date, dans Biggar, Les Précurseurs, 60. 
différentes. Car ils n'auraient pu se donner rendez-vous sur une terre qui n'était pas encore découverte.

La distance de la terre neuve varie aussi selon qu'on se fie à Cantino ou à Pasqualigo: le premier donne 2800 milles, ce qui est plus proche de la vérité, et le second réduit ce chiffre à 1800 milles, ce qui est insuffisant. Enfin, les deux agents italiens ne s'accordent pas sur le nombre des Indiens que le navire emportait à Lisbonne, en octobre 1501. Ils auraient été cinquante, selon Cantino; mais ce chiffre est invraisemblable, si l'on considère la difficulté d'une telle capture et la petitesse du navire. Pasqualigo semble plus près de la vérité, lorsqu'il parle de sept personnes, hommes, femmes et enfants; il en annonce cependant cinquante qui doivent arriver sur le navire de Gaspar Corte-Real ${ }^{59}$. Si utiles que soient ces sources et si unanimes qu'on les trouve à propos de la substance de l'événement, on ne peut donc les utiliser qu'avec prudence aussitôt qu'on en vient aux précisions de détail. Elles sont toutefois irremplaçables. Les récits de Cantino et de Pasqualigo demeurent, avec quelques autres pièces portugaises contemporaines, les meilleurs témoignages qu'on possède sur la découverte de Gaspar Corte-Real.

\section{LA TERRE DES CORTE-REAL}

Quel endroit et quelle étendue de la côte nord-américaine Gaspar Corte-Real a-t-il explorés en 1501 ? Il n'est pas douteux que le littoral oriental de Terre-Neuve soit compris dans cette exploration. Les descriptions que nous livrent Cantino et Pasqualigo, surtout le premier, conviennent à Terre-Neuve, si l'on atténue quelque peu leurs couleurs enthousiastes. Mais elles manquent de précisions topographiques. L'ensemble des cartes portugaises produites entre 1502 et 1530 fournit toutefois un témoignage global beaucoup plus décisif: la Terre des Corte-Real correspond sans conteste à la côte orientale de TerreNeuve. Ce point paraissant acquis, on doit poser de nouveau la seconde partie de la question: quelle étendue de la côte terreneuvienne Gaspar Corte-Real a-t-il parcourue ? D'après Pas-

59 “... et cum altra caravella che se aspecta d'hora inhora ne vien -1tri cinquanta" (Lettre du 19 octobre, Harrisse, Les Corte-Real..., 212). 
qualigo, il aurait côtoyé plus de six cents milles de littoral ${ }^{60}$. Ces milles, qui sont vraisemblablement des milles romains, à raison de quatre milles à la lieue, donneraient cent cinquante lieues. Mais Pasqualigo n'est pas un témoin de tout repos, quand il descend à ces détails. C'est lui qui donne mille huit cents milles de distance entre Terre-Neuve et Lisbonne, ce qui ne fait pas le compte.

Force est de recourir à la cartographie. Mais on entre alors dans une jungle où beaucoup d'esprits alertes se sont égarés. La critique des cartes du premier quart du seizième siècle, du moins en ce qui concerne l'Amérique du Nord, est extrêmement difficile. Contentons-nous ici d'une appréciation globale. Ces cartographes sont des artistes qui travaillent en cabinet, la plupart du temps. Leurs sources ne sont pas des relevés techniques, mais des informations recueillies de la bouche des explorateurs ou des marins. Cela ne vaut d'ailleurs que pour les cartographes soucieux ou capables de s'informer directement. Le plus souvent, on recopie des cartes plus anciennes, on en compose plusieurs ensemble, on les interprète et on crée même parfois. On peut noter, comme loi générale de l'évolution cartographique, que les premiers et les plus scientifiques de ces dessinateurs représentent séparément les diverses informations reçues. La carte appelée Cantino en est un exemple et aussi la carte de Freducci, à propos de notre continent. Mais l'opinion générale étant que tous ces pays nouveaux appartenaient à un même continent, qu'on l'appelât ou non l'Asie, les cartographes secondaires, surtout les vulgarisateurs, ont eu tendance à relier et à composer les informations en une seule représentation continue. Le procédé comporte évidemment beaucoup d'interprétation prématurée, comme on le voit déjà sur la carte de Ruysch, en 1507/1508. Le plus grave est que ces interprétations gratuites sont à leur tour objet de spéculation et d'interprétation. En fait, alors même que la cartographie se forge une méthode plus exacte et plus rigoureuse, la représentation de l'Amérique du Nord, après un demi-siècle de croissance, se

60 Biggar, Les Précurseurs, 65. 
dégrade de plus en plus jusqu'à la fin du seizième siècle, faute de vérification sur les lieux.

Le dessin qui durant ce siècle a servi de base à la représentation de notre littoral atlantique, au nord du cap Breton, est celui des cartes portugaises, entre 1502 et 1530. Son élément principal est une côte orientée directement du sud au nord et regardant vers l'est, où ne se trouve reproduite que très grossièrement la physionomie de Terre-Neuve. Sa longitude est à l'est de la ligne de Tordesillas ${ }^{61}$, c'est-à-dire trop rapprochée de l'Europe de quelque six degrés. Sa longueur n'est pas moins exagérée. L'extrémité sud tend à se rapprocher du $47^{\circ}$ degré de latitude nord, qui est la hauteur approximative du cap de Raze. Mais l'extrémité nord oscille entre le $55^{\mathrm{e}}$ degré et le $60^{\mathrm{e}}$, ce qui est manifestement trop haut. D'ailleurs, sur la plus grande partie d'une telle hauteur, la côte de l'Amérique ne se dirige pas du sud au nord, mais elle incline fortement au nord-ouest. Il y a aussi de fortes raisons de suspecter les mesures de la côte méridionale de Terre-Neuve sur ces cartes. En sorte qu'on ne peut guère faire fond sur un tel dessin pour une discussion des découvertes des Corte-Real.

En l'absence de témoignages dignes de foi à ce propos, le fil conducteur le plus approprié sera la toponymie la plus ancienne de Terre-Neuve. Entre 1502 et 1540, la cartographie

${ }^{61}$ Le 4 mai 1493, par la bulle Inter cætera divinæ maiestati, le pape Alexandre VI donnait à la Castille toutes les terres inconnues situées à l'ouest d'un méridien qu'il précisait ainsi: "quæ linea distet a qualibet insularum, quæ vulgariter nuncupantur de los Azores y Cabo Verde, centum leucis versus occidentem et meridiem...". Le roi du Portugal, assez peu content de cette décision, négocia avec les souverains d'Espagne et l'on arriva à une entente, le 2 juillet 1494 , à Tordesillas. Le traité de Tordesillas, comme on l'appelle, reportait le méridien à deux cent soixante-dix lieues plus à l'ouest, c'est-à-dire à trois cent soixante-dix lieues à l'ouest des îles mentionnées plus haut. A cause de l'imprécision des termes employés, les deux pays disputeront indéfiniment sur l'exacte situation de cette ligne. Mais si l'on compte, selon Pierre Crignon (Ramusio, Navigationi et Viaggi..., (Venise, 1556), III : 423) dix-sept lieues comme largeur d'un degré de longitude dans la région équinoctiale, ce méridien ne peut pas se trouver plus à l'ouest que le $47^{\mathrm{e}}$ degré de longitude ouest de Greenwich. Or le point le plus oriental de Terre-Neuve est environ au $52^{e}$ degré de la même longitude, c'est-à-dire au moins cinq degrés trop à l'ouest pour se trouver en zone portugaise. Les premiers cartographes portugais ont dû avancer la terre de Corte-Real d'au moins cette distance vers l'est, pour la faire entrer dans le domaine portugais. 
rapporte une nomenclature abondante pour le littoral délimité plus haut. Elle est tout entière d'origine portugaise, le Portugal étant alors le seul pays à produire des cartes originales et autorisées de cette partie de notre continent. D'autre part, dès 1534, Jacques Cartier est témoin qu'il existait aussi une toponymie française sur les mêmes côtes. Et son témoignage se trouve corroboré et enrichi par deux autres cosmographes français contemporains, Pierre Crignon et Jean Alfonse. La confrontation de ces sources va permettre de dresser un argument toponymique dont la signification, espérons-nous, ne manquera pas d'être concluante.

Il faut remarquer d'abord que les documents nous fournissent deux sortes de noms géographiques. Il y a ceux dont les cartographes parsèment leurs cartes et il y a ceux par lesquels les marins désignent les endroits qu'ils visitent. Appelons les premiers noms cartographiques et distinguons les seconds par l'épithète d'usuels.

Le plus souvent, les cartographes ont emprunté leur toponymie aux rapports des découvreurs et des explorateurs, parfois aussi aux marins habitués des lieux. Ces derniers, toutefois, faisaient d'ordinaire difficulté à livrer leurs secrets. En sorte que la cartographie transmet surtout la toponymie des découvreurs. Il ne suffit cependant pas qu'un nom ait été apposé sur un lieu par un explorateur pour qu'il s'y établisse à demeure ${ }^{62}$, il y faut aussi un usage traditionnel des marins. Si le lieu n'est pas aussitôt fréquenté, la toponymie va se déplacer, se corrompre ou se perdre ${ }^{63}$. Ainsi en est-t-il arrivé de toute la nomenclature de Verrazano et d'une grande partie de celle de Cartier. De plus, les méthodes cartographiques de l'époque,

62 On pourrait citer une foule de noms qui ne sont plus sur leur lieu d'origine, grâce aux manipulations des cartographes. Enumérons seulement les plus connus: Labrador, Saint-Laurent, Acadie, Canada.

63 C'est grâce à une erreur de Ramusio, en 1556, que le nom de SaintLaurent est passé d'une petite baie sur la rive nord du golfe à tout ce même golfe. Si la baie de Saint-Laurent nommée par Cartier avait été assidûment fréquentée depuis 1534, l'erreur du cosmographe n'aurait pu prévaloir. On peut dire la même chose du nom de Sainte-Croix, donné par Cartier à notre rivière Saint-Charles et qui a fini par se fixer à Sainte-Croix de Lotbinière. 
consistant à reproduire indéfiniment des sources trop imprécises, à les composer et à les interpréter de diverses façons, étaient mortelles pour la toponymie. Les noms étaient rapidement défigurés; ils se déplaçaient; les appellations d'origines diverses se trouvaient mélangées et on les intervertissait le plus facilement du monde. La toponymie cartographique de l'Amérique du Nord, au seizième siècle, est foncièrement instable.

Les marins ont besoin de noms pour désigner les principaux points d'un littoral qu'ils fréquentent. Ces noms ils les créent le plus souvent eux-mêmes, s'ils ne préexistent pas. Et aussitôt qu'ils ont commencé à se les transmettre de l'un à l'autre, les vocables ne peuvent plus changer radicalement, bien qu'ils puissent parfois être corrompus. D'autres fois, les marins empruntent les noms aux découvreurs, surtout si la fréquentation des lieux suit de près la découverte ou si le découvreur a laissé de bonnes descriptions. Mais si les cartographes servent d'intermédiaires entre le découvreur et les pilotes, la toponymie court des risques. Il suffit d'un intervalle de peu d'années pour que la toponymie d'un découvreur se transforme sur les cartes ${ }^{64}$. Certains noms ont eu une grande notoriété avant d'être fixés par les marins sur les lieux mêmes ${ }^{65}$. Mais, dans la plupart de ces derniers cas, la localisation finale du nom n'est pas celle qui lui avait été primitivement destinée. Une fois que la tradition toponymique usuelle est établie en un lieu, elle ne change plus en substance, même s'il peut y avoir des altérations accidentelles. Le caractère principal d'une toponymie usuelle est la stabilité. Et c'est pourquoi elle finit toujours par triompher d'une toponymie cartographique différente, même plus longuement et plus largement diffusée.

Sous le rapport de la toponymie, la côte nord-américaine des premières cartes portugaises peut être divisée en trois sections. Nous les énumérons du sud au nord: $1^{\circ}$ la côte méri-

64 Un des exemples les plus significatifs est celui du cap de Lorraine, nommé par Jacques Cartier, qui a eu le temps d'être changé en cap Saint-Laurent avant d'être fixé par une tradition maritime.

65 C'est ainsi que Labrador, repoussé vers l'ouest par l'ancien nom, Groenland, a fini par désigner, dans le langage maritime, le littoral qui est au nord du détroit de Belle-Isle. 
dionale de Terre-Neuve, du cap Breton au cap de Raze; $2^{\circ}$ la côte orientale de Terre-Neuve, depuis le cap de Raze jusqu'à l'île Fogo; $3^{\circ}$ le reste de la même côte orientale, à partir de l'île des Oiseaux en montant vers le nord. Nous mettrons en regard deux traditions toponymiques, française et portugaise, telles qu'on les trouve attestées dès avant 1540, c'est-à-dire au temps où toute la cartographie nord-américaine est encore exclusivement inspirée par les Portugais. La tradition française sera représentée par des auteurs français, Jacques Cartier, Pierre Crignon, Jean Alfonse. En regard de ces deux listes, nous présenterons une liste des noms modernes correspondants, tels qu'il se sont conservés.

\section{Première section}

Cap Breton

Iles de Saint-Pierre 66

Cap de Sainte-Marie 68

Port de Saint-Christophe 70

Cap de Chincète 72
Cap Breton

Arcipelago das 11000 Virgens 67

Cabo de S. Paulo 69

Porto da Cruz ${ }^{71}$
Iles de Saint-Pierre et Miquelon

Cap de Sainte-Marie

Baie de Sainte-Marie Cap Pine (de Pène) et Saint Shotts (Chincète)

66 En 1536, Jacques Cartier trouva ce nom déjà en usage à cet endroit, après avoir longé la partie ouest de ce rivage sans y avoir relevé de toponymie, ni aucun signe d'occupation. A Saint-Pierre, cependant, il vit "plusieurs navires, tant de France que de Bretaigne" (Biggar, The Voyages of Jacques Cartier (Ottawa, 1924), 239).

67 Le nom des Onze-mille-Vierges a été créé par Jean Alvares Fagundes, vers 1520. Il apparaît pour la première fois, autant qu'on sache, sur la carte Miller, puis sur la carte Maggiolo, de 1527. Il sera le nom cartographique le plus marquant de cette rive. Déplacé sur les cartes par l'apparition du nom de Saint-Pierre, après 1540, il finira par se fixer plus à l'ouest, sous la forme presque méconnaissable de Burgeo.

${ }^{68} \mathrm{Le}$ nom est mentionné, sur son site actuel, par Jean Alfonse (Biggar, The Voyages of Jacques Cartier, 300).

$69 \mathrm{Ce}$ nom semble avoir désigné le cap Sainte-Marie sur les cartes portugaises. Il disparaîtra entièrement à l'apparition des noms français.

70 Ce port, dont le nom n'a pas subsisté, semble être la baie de SainteMarie. Il est vraisemblable que le nom si connu de Sainte-Marie, donné au cap voisin, l'aura supplanté pour désigner la baie.

71 Porto da Cruz accompagne d'ordinaire le cabo de S. Paulo sur les cartes portugaises. Il a pu désigner la baie Sainte-Marie. Peut-être aussi s'agirait-il de la baie des Trépassés, plus à l'est. En tout cas, il a disparu, laissant la place aux noms français.

$72 \mathrm{Ce}$ nom est mentionné par Jean Alfonse. Il n'est pas douteux qu'il ait désigné le cap Pine (corruption du nom français Pène, al. Pane). Il demeurait à cet endroit au dix-septième siècle et il semble bien qu'on le lise encore sous la corruption anglaise de Saint Shotts. 


\section{Deuxième section}

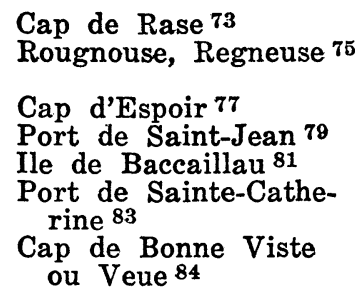

Iles de Feu 88

$\begin{array}{ll}\text { Cabo Raso 74 } & \text { Cap de Raze (Race) } \\ \text { Farilhâo 76 } & \text { Renewse } \\ \text { Cabo de Spera 78 } & \text { Cap Spear } \\ \text { Baia de S. Joham 80 } & \text { Saint-Jean } \\ \text { Ilha dos Bacalhaos } 82 & \text { Ile de Baccalieu }\end{array}$

Catalina

Cabo de Bôaventura 85 Cap Bonavista

Ilha de Frey Luis 86

Cabo do Marco 87

Illa do Fogo 89
Cap Freels

Ile Fogo

${ }^{73}$ C'est la forme la plus fréquente en français. Cap Ras serait une meilleure traduction du nom portugais. Cartier, Crignon et Alfonse ir mentionnent.

${ }_{74}$ Cabo Raso n'est probablement pas un nom qui remonte à CorteReal lui-même. Il n'est pas sur les plus anciennes cartes. Mais il apparaît à une époque reculée, apparemment créé par les pêcheurs portugais. Il est sur la carte Add. MS 31316 du British Museum et sur le prototype Freducci. C'est le nom qui montre la plus grande stabilité sur les cartes, ce cap étant le principal point de repère pour les marins venant d'Europe. 75 Excellent exemple d'une corruption française pour Ronhosa, la Malicieuse. Cartier est témoin de ce nom, en 1536. Alfonse écrit Rogneuse. Les Anglais rendent le mot par Renewse.

76 Farelhão, petit promontoire, îlot escarpé. Le mot est déjà attesté par la carte Vallicelliana. Les Français écriront Farillon et les Anglais nomment aujourd'hui ce point Ferryland.

77 Le nom se trouve dans la Cosmographie de Jean Alfonse, simple traduction du mot portugais. Le mot anglais, Spear, est une pure corruption. 78 Nom qui est aussi très ancien, mais qui est une création des pêcheurs plutôt que de Corte-Real. On le voit sur la carte Oliveriano-Pesaro et sur le prototype Freducci.

79 Jean Alfonse est témoin pour ce nom, qui était bien connu des Français. Cartier s'arrêtera dans cette baie en 1542.

$80 \mathrm{~S}$. Joham apparaît sur la carte Miller, après 1520. C'est là que Gilbert prendra possession de Terre-Neuve au nom de la reine Elisabeth, en 1583.

81 Jean Alfonse mentionne cette île.

$82 \mathrm{Ce}$ nom de Bacalhaos est probablement le plus ancien qu'ait suscité la découverte des pêcheries à Terre-Neuve. On le trouve déjà, quoique défiguré, sur la carte de Ruysch, de 1507-1508. La richesse de Terre-Neuve en poissons fut révélée aux Portugais par Corte-Real en 1501, et c'est ce qui amena les Bretons dans le voisinage dès 1504. L'île de Bacallieu marqua probablement le premier lieu de pêche des Portugais.

${ }^{83} \mathrm{Le}$ port de Sainte-Catherine est le lieu de l'atterrage de Jacques Cartier, en 1534. Le nom s'est perpétué sous sa forme ibérique, Catalina. 84 Jacques Cartier, en 1534, traduisait Bonne Viste. Le texte italien attribué à Pierre Crignon porte Bonavista. Jean Alfonse écrivait Bonne Veue. Ces traductions des Français prouvent que la tradition était établie chez les pêcheurs portugais de dire Bóavista. 


\section{Troisième section}

Ile des Oiseaux 90

Belles-Isles 92

Cap Dégrat 94

Ile de Karpon 96

Belle-Isle 98

Baie des Chasteaux 100

\author{
Ilha das Aves 91 \\ Ilhas de S. Joham \\ et $\mathrm{S}$. Pedro 93 \\ Cabo do Marco 95 \\ Ilha do Tormento 97 \\ Ilha da Fortuna 99 \\ (Détroit) 101
}

\author{
Ile Funk \\ Iles Groais et Bell \\ (Belle-isle du Sud) \\ Cap Dégrat \\ Ile de Querpont \\ Belle-Isle \\ Détroit de Belle-Isle
}

85 En dépit de la tradition locale, les cartes écrivent Bõaventura (Bonne Chance), au lieu de Bõavista (Bonne Vue). Il s'agit évidemment du même lieu. Il semble bien que les cartographes maintiennent ici le nom donné par le découvreur, Gaspar Corte-Real, bien que les pêcheurs aient très tôt corrompu le mot en Bõavista, qui désigne encore ce cap célèbre.

$86 \mathrm{Ce}$ nom de Frey Luis est déjà sur la carte Kunstmann III. Mais on ne saurait dire s'il remonte jusqu'à Corte-Real. Il désignait une île qui se trouvait probablement dans la baie de Bonavista. On ne peut assurer absolument qu'il s'est perpétué. Mais une carte anglaise de 1710, celle de Senex et Maxwell, appelle la baie de Bonavista B. de Frelay. Et nous croyons que le nom est passé au cap voisin sous la forme Freels. Car ce cap, ayant perdu son appellation do Marco qui était peut-être purement cartographique, paraît avoir été longtemps sans nom.

87 Le cabo do Marco, inscrit sur notre cap Freels, est antérieur à 1520. Il apparaît déjà sur la carte Oliveriano-Pesaro et sur le prototype Freducci.

88 Jean Alfonse nomme ainsi l'île Fogo avec celles qui l'entourent: les îles de Feu.

${ }^{89}$ L'île Fogo est mentionnée par le prototype Freducci. Il s'agit probablement d'une extension des pêcheries portugaises entre 1510 et 1520; car ces parages sont dangereux pour la navigation et on ne devait pas s'y aventurer volontiers. Les cartes mentionnent aussi un rio das Gamas en conjonction avec cette île. Ce ne peut être que le fleuve Gander, qui se jette à proximité. Rio das Gamas signifie fleuve des Daines.

${ }^{90}$ L'île des Oiseaux est un nom qu'on trouve sous la plume de Cartier, en 1534. Jean Alfonse l'appelle l'île des Apponas, un nom d'oiseau emprunté à Jacques Cartier. Ici, c'est le français qui est original; le portugais sera une traduction. Cartier et Alfonse laissent bien voir quelle importance a cette île pour la navigation vers le détroit de Belle-Isle.

91 Les mentions apparemment les plus anciennes se trouvent sur la carte Miller et sur la carte Pedro Reinel, après 1520.

92 Les Belles-Isles, mentionnées par Cartier en 1534 et aussi par Jean Alfonse, sont l'île de Groais et celle de Belle-Isle du Sud, deux îles importantes pour la navigation vers le détroit de Belle-Isle.

93 San Joham et Sam Pedro, qui est aussi un couple onomastique de Fagundes, occupe la position et le rôle des Belles-Isles sur les cartes portugaises, à commencer par les cartes Miller et Pedro Reinel. Leur situation, avant un cap au nord duquel se trouve un détroit, ne peut pas tromper.

94 Cartier parle de havre du Dégrat en une île où s'élève un cap élevé, qui est le cap Dégrat.

95 Le cabo do Marco est le deuxième cap de ce nom. Celui qui était situé sur le cap Freels était plus ancien. Celui dont il s'agit ici fait partie 
Comme on le voit, la liste des noms d'origine portugaise est celle qui a subsisté dans la deuxième section. La liste française correspondante n'en est que la traduction et elle se ressent de l'origine portugaise des mots. En cet endroit, la toponymie des cartographes a coïncidé, au moins en gros, avec la toponymie qu'employaient les marins portugais sur les côtes de TerreNeuve. Il est vrai que les cartographes embrouillent volontiers l'ordre de ces noms; mais la mention de ceux-ci à une époque aussi reculée, confirmée encore par leur persistance sur le même rivage, nous assure que leur ordre véritable n'est pas celui de la cartographie, mais celui de la géographie actuelle. C'est parce qu'elle a été dès lors usuelle, et non parce que les cartographes lui ont fait une propagande, que cette toponymie s'est perpétuée jusqu'à nos jours. Les Français l'ont trouvée toute faite et ils n'ont eu qu'à l'adopter, soit en la traduisant, soit en lui imprimant leur propre accent. Les premiers à créer des appellations sur cette côte et à se les transmettre d'un voyage à l'autre ont done été des Portugais.

du groupe île das Aves, îles S. Joham et $S$. Pedro et îles do Tormento et da Fortuna. Carpon.

96 Cartier mentionne cette île de Karpont, en 1534. Alfonse écrit

97 L'identification de l'ilha do Tormento avec le Querpont est conjecturale. C'est que cette dernière île semble la seule, dans la région, à avoir assez d'importance pour être mise, conjointement avec Belle-Isle, en rapport avec les noms do Tormento et da Fortuna.

98 Belle-Isle, parfois appelée du Nord, est mentionnée par Cartier et Alfonse, au milieu du détroit qui porte aujourd'hui ce nom.

${ }^{99}$ L'ilha da Fortuna qui jumelle l'ilha do Tormento sur les cartes portugaises est ordinairement dans une position qui provoque cette identification avec Belle-Isle.

100 La baie des Chasteaux est nommée par Jacques Cartier, en 1534, par Pierre Crignon, en 1539. Elle désigne, chez Cartier, soit une baie de la rive nord du détroit où le nom subsiste aujourd'hui, soit le détroit lui-même. Crignon entend le détroit par ce nom. Jean Alfonse appelle le détroit la Grand Baye.

101 Nous ne doutons pas que le détroit indiqué au nord des cartes portugaises soit le détroit de Belle-Isle, que Cartier et Crignon appellent baie des Chasteaux. Il importe peu que le détroit portugais soit trop élevé en latitude pour correspondre à la latitude du détroit de Belle-Isle. La mappemonde harléienne a manifestement fait cette identification entre la baie des Chasteaux et le détroit portugais, au soixantième degré de latitude ! (v. planche VIII, dans Biggar, The Voyages of Jacques Cartier, après la p. 128). Les anciennes cartes portugaises montaient toutes beaucoup trop haut et les premières cartes françaises les ont imitées. 
Nous aurions pu citer plusieurs autres noms d'origine portugaise qui se sont perpétués sur le même littoral. Ils remontent certainement jusqu'à cette époque reculée. On les aurait tous intercalés dans la liste de la deuxième section. Nous avons fait choix des noms les plus anciennement attestés, en quantité suffisante pour illustrer notre propos.

Dans les deux autres sections de Terre-Neuve, c'est exactement le phénomène inverse que l'on constate. C'est la liste française qui a survécu et tous les noms portugais des vieilles cartes se sont évanouis. Et cela en dépit du fait que ces cartes n'ont fait de publicité qu'aux seuls noms portugais jusqu'en 1540 au moins. La liste française y a si vigoureusement supplanté la liste portugaise que les cartes produites postérieurement au Portugal et en Espagne l'ont adoptée. La signification de ce fait est assez évidente. Les Français n'ont pas trouvé, au nord de l'île Fogo et au sud de Terre-Neuve, une toponymie portugaise déjà établie sur les lieux. Autrement, ils l'auraient adoptée, comme ils l'ont fait de celle de la côte nord-sud, du cap Freels au cap de Raze. Ainsi, ils ont été les premiers à créer une tradition toponymique usuelle dans ces deux sections et celle que rapportaient au même temps les cartographes européens était purement cartographique.

L'extension d'une toponymie portugaise au sud de TerreNeuve et au nord de l'île Fogo n'a d'ailleurs eu lieu qu'à une époque relativement tardive. Si l'on ne considère que les noms qui vont subsister, tous ceux qui apparaissent avant 1520 appartiennent à notre deuxième section: Frey Luis (Freels), Concepcion (baie de la Conception) ${ }^{102}$, Raso (cap de Raze), Bacalhaos (île Baccalieu), Bõaventura (Bonavista), Spera (cap Spear). D'autres noms, disparus très tôt, appartenaient aussi à cette section; car ils sont intercalés entre ceux qui ont eu plus de succès: Santa Cyria (corruption de Santa Iria: baie de

$102 \mathrm{Ce}$ nom aurait pu prendre place dans la liste portugaise formée plus haut, deuxième section. Il apparaît déjà sur les cartes Kunstman III et Freducci. Il pourrait même avoir Corte-Real pour auteur. 
la Trinité) ${ }^{103}$, rio da Rosa (fleuve au sud de Bonavista) ${ }^{104}$, Medões (les Terreurs, apparemment les rochers de la baie de Bonavista) ${ }^{105}$. Et surtout un cabo do Marco, un cap de la Démarcation (celle de Tordesillas) qui désigne certainement une ancienne limite septentrionale du territoire portugais et qui est placé sur le cap Freels ${ }^{106}$. En sorte que les plus anciennes cartes portugaises ne représentaient pas un territoire plus étendu que celui qui est compris entre le cap Freels, au nord, et le cap de Raze, au sud. La carte de Freducci nous a conservé le dessin de cette section, telle que les Portugais la voyaient avant qu'on étendît une toponymie au sud de Terre-Neuve et au nord de l'île Fogo ${ }^{107}$. La liste de ces appellations, lues du nord au sud, décrit le littoral mieux que ne le ferait une carte: rio das Gamas (fleuve Gander), ylha de Fuego (île Fogo), cabo do Marco (cap Freels), monte de Trigo et ylha de Frey Luixi (dans la baie de Bonavista), ylha de Becaliab (île Baccalieu), baia de Concepçam (baie de la Conception), cabo Desperado (cap Spear), cabo Raxo (cap de Raze).

On peut comparer plusieurs cartes postérieures à 1520, Miller I, Anonyme-Weimar 1527, Pedro Reinel, Verrazano 1529, Anonyme-Lange, avec la carte Freducci ${ }^{108}$. On constatera qu'elles ont toutes visiblement ajouté au dessin Freducci une grande baie, au nord de Fogo, pour contenir un groupe additionnel de noms et que cette baie est bornée au nord par un détroit qui n'apparaisait pas sur les cartes plus anciennes. Les noms ajoutés, selon les cartes où la liste est plus complète, sont les suivants du sud au nord: ilha das Aves, les îles jumelles de $S$. Pedro et S. Joham, un second cabo do Marco qui double le premier et plus ancien, deux îles dans le détroit, do Tormento

${ }^{103}$ Santa Iria (Sainte-Irène) est ce qu'il y a de plus authentiquement portugais. On retrouve ce nom dans Cova da Iria et dans Santarem.

104 Nous dirons plus loin un mot sur ce fleuve.

105 Appelés aussi Baxos do Medo sur la carte Kunstmann III.

106 Cela est particulièrement évident sur la carte Freducci, dont nous parlons un peu plus bas.

${ }^{107} \mathrm{Au}$ nord de Fogo, la côte n'est pas continuée. Au sud, la côte est dessinée, mais sans toponymie.

${ }_{108}$ La fig. 19 (p. 73), dans B. G. Hoffman, Cabot to Cartier, illustre fort bien cette comparaison. 
et $d a$ Fortuna. Il s'agit évidemment d'un ensemble de repères maritimes pour la navigation jusqu'à Belle-Isle, dont le détroit est montré comme borne septentrionale de tout ce territoire.

On sait en effet qu'au détour du cap Freels, le plus ancien Marco portugais, la mer est parsemée de rochers dangereux. Les voyageurs à destination de Belle-Isle doivent naviguer directement au nord quelque trente milles, avant de mettre le cap sur le détroit. Ils arrivaient alors à ce qui est aujourd'hui l'île Funk. C'est l'ilha das Aves des cartes portugaises, qui indiquait ainsi la direction du détroit et fournissait en surplus un abondant ravitaillement en chair d'oiseaux. L'ilha das Aves était pour les marins la clef du détroit de Belle-Isle et le fait que les Portugais ne l'aient pas marquée sur leurs plus anciennes cartes est une nouvelle indication qu'ils n'étaient pas encore allés aussi loin. De l'île des Oiseaux on ne se dirigeait pas immédiatement à l'île de Querpont, à l'extrémité nord de Terre-Neuve; c'eût été risquer de passer au large et de la manquer. On s'adressait à deux îles qu'on appelle aujourd'hui Groais et Belle-Isle du Sud (Bell). Les cartes portugaises nomment la première San Joham et l'autre Sam Pedro. De ces îles on est déjà en vue du rivage de Terre-Neuve, que l'on suit en montant vers le nord, guidé par la hauteur du cap Dégrat. Ce dernier point est celui qui portait le nom portugais do Marco, le second cap de ce nom. Contournant ce cap et l'île de Querpont, probablement appelée do Tormento, on entrait dans le détroit, au milieu duquel on voyait Belle-Isle, désignée sur les cartes comme ilha da Fortuna. Cette route est celle qu'a suivie Jacques Cartier en $1534^{109}$ et que Jean Alfonse va décrire quelques années plus $\operatorname{tard}{ }^{110}$.

Nous considérons comme certain que la baie des Chasteaux de Cartier et de Crignon, appelée Grand Baye par Jean Alfonse, n'est pas autre chose que le détroit septentrional des cartes portugaises produites entre 1520 et 1530 et que tout cela n'est qu'une seule réalité géographique appelée aujourd'hui détroit

109 Biggar, The Voyages of Jacques Cartier, 11-12.

110 Ibid., 280-283. 
de Belle-Isle. Ce point est d'une particulière importance pour assurer le bien-fondé des vues précédemment exposées. Aussi nous y attarderons-nous quelque peu. Peu importe que l'ancien détroit portugais ait été situé entre le $55^{\mathrm{e}}$ et le $60^{\mathrm{e}}$ degré de latitude nord, tandis que le détroit de Belle-Isle n'est qu'au 51. Cette divergence embarrassera plusieurs cartographes postérieurs à 1540, lorsqu'ils voudront représenter les explorations de Cartier. Mais nous avons un témoin qui est très rapproché du découvreur malouin et qui a fait cette identification dès après le premier voyage de ce dernier, en 1534. Ce témoin est un prototype cartographique qui a servi de source commune à deux documents très connus: la carte de Jean Roze ${ }^{111}$ et la carte harléienne ${ }^{112}$. Ces deux cartes, dont on fait d'ordinaire remonter trop haut la date, n'ont probablement été dessinées qu'après 1540. Il est évident que leur dessin du golfe reproduit celui d'une carte où l'on avait utilisé les résultats de l'exploration de 1534, y compris les erreurs que Cartier commit cette année-là et qu'il corrigea en 1535-1536. Malgré les retouches que les deux cartographes postérieurs ont fait subir à son dessin, on perçoit très bien que l'auteur du prototype de $c a$ 1535, parlant portugais et usant d'une technique apparentée à celle des Homem, ne connaissait pas encore l'insularité de l'île d'Anticosti, ni celle des îles de la Madeleine, ce qui ne pourra plus être ignoré après 1536 . Or ce prototype portugais, très bien renseigné sur les découvertes de Cartier dès 1535, identifie clairement la baie des Chasteaux de Cartier avec le détroit des anciens Portugais, tout en la plaçant, selon Roze, au $55^{\mathrm{e}}$ degré ou, selon la carte harléienne, au $60^{\mathrm{e}}$. Ce témoignage d'un contemporain remarquablement averti, conjugué avec les convergences et les vraisemblances déjà signalées, devrait suffire à notre propos. La baie des Chasteaux, ou détroit de Belle-Isle, et l'ancien détroit portugais coïncident l'un avec l'autre.

En bref, il ressort de cette analyse que la plus ancienne occupation portugaise a été jusqu'en 1520 bornée au sud par

111 Reproduite dans Biggar, The Voyages of Jacques Cartier, pl. V, après la p. 64 .

112 Ibid., pl. VIII, après la p. 128. 
le cap de Raze et au nord par le cap Freels, sur lequel on a fixé un premier Marco. Au-delà de ces limites au sud, la toponymie révèle une occupation originellement française, depuis le cap de Raze jusqu'aux îles de Saint-Pierre-et-Miquelon. Au nord du cap Freels ou de l'île Fogo, la toponymie porte encore témoignage en faveur d'une première occupation française. Cependant, après 1520 , la cartographie, encore toute d'inspiration portugaise, réclame pour la couronne du Portugal tout le nord de Terre-Neuve, posant un second Marco au cap Dégrat, de même que la côte méridionale de la grande île, depuis le cap de Raze jusqu'à l'archipel des Onze-mille-Vierges (SaintPierre-et-Miquelon).

Les considérations qui précèdent inciteront peut-être à porter plus d'attention au témoignage du Gran Capitano de Ramusio, dont Pierre Crignon fut le rédacteur. Ce capitaine français, dont le témoignage fut rédigé en 1539, ne connaissait encore rien des découvertes de Jacques Cartier. Il ignorait donc tout du caractère insulaire de Terre-Neuve. Son information était en partie tirée des cartes alors en circulation. Mais il avait des connaissances qui ne relevaient pas de ces sources. Ses longitudes, différentes de celles des Portugais, étaient encore plus inexactes. Mais ses latitudes étaient à peu près justes. Il était bien renseigné sur les habitants et les productions du pays, comme quelqu'un qui a recueilli ses renseignements de témoins expérimentés. Il ne paraît cependant pas avoir lui-même voyagé dans cette partie du nouveau continent. Écoutons ce qu'il dit des découvertes.

La dite terre (la Terre-Neuve) a été découverte depuis trente-cinq ans ençà, c'est-à-dire cette partie qui court levant et ponent, par les Bretons et les Normands. Pour cette raison cette terre est appelée le cap des Bretons. L'autre partie, qui court nord et sud a été découverte par les Portugais, depuis le cap de Ras jusqu'au cap de Bonavista. Elle contient environ soixante-dix lieues. Et le reste a été découvert jusqu'au golfe des Châteaux, et plus outre, par lesdits Bretons et Normands. Il y a environ trentetrois ans qu'un navire de Honfleur, dont Jean 
Denys était capitaine, avec le pilote Camart de Rouen, y alla pour la première fois. Et en l'année 1508, un navire de Dieppe appelé La Pensée, qui appartenait à Jean Ango, père de Monseigneur le Capitaine et Vicomte de Dieppe, y alla, Thomas Aubert étant maître ou patron dudit navire; et il fut le premier qui conduisit ici des gens dudit pays ${ }^{113}$.

Le témoignage rapporté par Ramusio est évidemment profrançais, mais pas davantage que les cartes de l'époque ne sont pro-portugaises. Le marin normand sait d'ailleurs que l'on conteste à son temps une découverte française de la "Terre neuve", parce qu'il remarque, un peu plus loin à propos du rivage exploré par Verrazano, que les Portugais eux-mêmes concèdent cette dernière partie à la France ${ }^{114}$. Reste que son témoignage complète celui des cartes et se trouve encore confirmé par elles, non moins que par la tradition géographique ultérieure. Il l'est de nouveau par un témoignage indépendant de 1512, rapportant l'arrivée d'indigènes américains à Rouen en $1508^{115}$. La suggestion la plus saugrenue que Ganong, écrivain qui a par ailleurs droit à beaucoup d'admiration, ait jamais faite est celle où il propose d'entendre le mot "Bretons" des cartes anciennes au sens de British plutôt que d'Armoricains ${ }^{116}$.

113 "Detta terra è stata scoperta da 35 . anni in qua, cioè quella parte che corre levante \& ponente, per li Brettoni \& Normandi, par la qual causa è chiamata questa terra il capo delli Brettoni. L'altra parte che corre tramontana \& mezzo dì è stata scoperta per li Portoghesi dopo il capo di Ras fino al Capo di buona vista, il che contiene circa 70. leghe, \& il restante è stato scoperto fin al golfo delli castelli, \& piu oltra, per detti Brettoni \& Normandi. \& sono circa 33, anni che un navilio di Honfleur, del quale era Capitano Giovanni Dionisio, \& il piloto Camarto di Roano, primamente vi ando, \& nell'anno 1508, un navilio di Dieppa detto la Pensee, il quale era gia di Giovan Ango padre del Monsignor lo Capitano \& Visconte di Dieppa vi ando, sendo maestro over patron di detta nave maestro Thomaso Aubert, \& fu il primo che condusse qui le genti del detto paese" (Pamusio, Navigationi et Viaggi, III : f. $423 \mathrm{E}-\mathrm{F}$ ).

114 "Questra terra da molti è detta la Francese, \& similmente per li Porthoghesi medesimi" (Ramusio, Navigationi et Viaggi, III, f. 423F).

115 Eusebii Casariensis Episcopi Chronicon (Paris, Estienne, 1512), f. 111. Cité par Henry Harrisse, Découverte et Evolution cartograp̉inique de Terre-Neuve et des Pays circonvoisins (Londres, 1900), 162-163.

116 W. F. Ganong, "Crucial maps in the early cartography and Placenomenclature of the Atlantic coast of Canada", I, Mémoires de la Société Royale du Canada (1929), II: 167-170. 
Cette proposition n'est soutenue par aucune source historique valable, alors qu'un faisceau imposant de convergences et de preuves appuie l'interprétation traditionnelle du terme Bretons. Les Anglais eux-mêmes, encore en 1521, confessent n'avoir ni maîtres ni mariniers anglais "exercised \& labored in the same parties, for to guyd there shipps \& other charges than we knowe of" 117. Les Bretons, au contraire, y sont si habitués dès 1511 que les étrangers songent à leur demander des pilotes. En octobre de cette année, la reine Jeanne de Castille envoie Jean d'Agramonte recruter des pilotes bretons pour le guider à TerreNeuve et elle lui permet de rapporter de Bretagne des vins, de la farine et de la viande ${ }^{118}$. Il s'agit bien de la Bretagne française, parce que l'Angleterre n'a jamais été un pays exportateur de vins.

Ce qui a fait le renom de Terre-Neuve, au seizième siècle, ce sont les pêcheries. Les noms portugais que nous avons relevés sur la côte orientale ont certainement eu pour auteurs les pêcheurs de morues qui balisaient ainsi leurs trajets. L'évolution cartographique porte cependant les traces d'une toponymie plus ancienne et qui est pour la plus grande partie tombée en désuétude dès les premières années de la fréquentation de TerreNeuve. L'auteur de cette toponymie primitive est bien probablement Gaspar Corte-Real. Bõaventura est l'un de ces noms. Il désigne probablement le cap où le découvreur s'est félicité de sa bonne chance d'apercevoir enfin un rivage inconnu. Le mot a conservé cette forme dans la cartographie primitive, les cartographes ayant une inclination particulière à perpétuer les vocables des découvreurs. Mais les pêcheurs portugais qui fréquentaient Terre-Neuve ont corrompu très tôt le nom en Bõavista, forme qui fut généralement acceptée et que les Français traduisirent par Bonne Viste ou Bonne Veue. Santa Iria, nom typiquement portugais, a été longtemps transmis dans la cartographie,

117 Biggar, Les Précurseurs, 137.

118 "Que por quanto vos aveis de yr por los pilotos, que con vos han de yr al dicho viaje, á Bretaña, que teneys voluntad de proveeros de alli de vinos é harina é carne, para lo traer al dicho puerto de Laredo ó Santander..." (Biggar, Les Précurseurs, 103). 
sans prendre racine dans la réalité. Il a été souvent corrompu en Cyria.

De même, il nous paraît assuré que le découvreur lui-même a nommé l'un des fleuves de Terre-Neuve rio da Rosa. Ce fleuve de la Rose est certainement le trait le plus marquant des plus anciennes cartes, celles d'avant 1510. Il y est situé à mi-chemin entre les deux extrémités de la côte de Corte-Real ${ }^{119}$. Cantino, premier rapporteur de l'expédition de Corte-Real, parlait en effet d'un fleuve que le découvreur avait remonté en naviguant sur la longueur d'une lieue ${ }^{120}$. La carte qui porte le nom du même Cantino représente ce trait géographique par un dessin en forme de trident pointé vers l'ouest. À cause de cette forme, on est tenté de l'identifier avec le Random Sound, phénomène géographique très remarquable dans la baie Trinité. Une telle interprétation pourrait aussi expliquer l'origine d'un nom obscur qui se lit sur la carte Kunstmann III: ilha emcorporada; car l'île Random, complètement encastrée dans celle de Terre-Neuve, s'y trouve vraiment incorporée. Ce fleuve, en même temps que son nom, a perdu sa signification par la suite, les pêcheurs ne s'intéressant qu'aux accidents maritimes du rivage. Le dessin de Cantino a cependant laissé des traces, qui se rapportent davantage au fleuve Gander après $1520^{121}$.

Dès 1501, Gaspar Corte-Real s'était rendu compte de la principale richesse de Terre-Neuve, les pêcheries. Pasqualigo, écrivant à ses frères le 19 octobre 1501, fait allusion à l'abondance des saumons, des harengs et des morues, qu'il appelle stochafis ${ }^{122}$. Les pêcheurs portugais n'ont pas tardé à exploiter

119 Ce trait est fortement souligné sur les plus anciennes cartes. Voir, par exemple, les fig. 11, 15 et 18 dans Hoffman, Cabot to Cartier, 58, 66 et 72 .

120 "... et correndo molti et grandi fiumi dolci per quella regione al mare, per uno de epsi forsi una legha fra terra intrarno, et in quella dismontati trovarno copia de suavissime et diversi fructi, et albori et pini ..." (Biggar, Les Précurseurs, 61).

121 Voir le tableau comparatif de la fig. 19, dans Hoffman, Cabot to Cartier, 73.

122 Stochafis est sûrement mis pour l'anglais stochfish, morue. Que ce mot anglais se trouve ici sous la plume d'un Italien écrivant du Portugal à des Italiens, voilà un fait non moins significatif que l'usage portugais du flamand bakeljauw. Pour les Méditerranéens, la morue était un poisson 
les pêcheries de Terre-Neuve. Et c'est apparemment la nouvelle de cette trouvaille qui a, dès 1504, attiré les Français en Amérique ${ }^{123}$. La vraie carrière de Terre-Neuve commence sûrement à l'île des Bacalhaos, l'île des Morues, petit rocher improductif, où l'on a découvert la première pêcherie. Ce nom, en tout cas, paraît sur les cartes de Maggiolo et de Ruysch, dès 1507/1508. Bacalhao n'est pas un mot d'origine latine, mais son usage courant est attesté au Portugal par un document de $1506{ }^{124}$. Il a certainement été emprunté au flamand, au temps où les Portugais allaient acheter la morue dans le nord de l'Europe. Le flamand désignait la morue par bakeljauw, de même racine que kabeljau allemand, qui avait donné au français $c a-$ billaud dès le moyen-âge. C'est par le portugais et grâce à la découverte de Terre-Neuve que ce vocable flamand est passé au seizième siècle dans les langues latines: bacallao en espagnol, baccala en italien, bacaliau en français. Les premières pêcheries portugaises furent probablement concentrées durant quelques années autour de l'île Baccalieu, comme on l'appelle aujourd'hui. Mais entre 1510 et 1520 , on dut en découvrir aussi autour de l'île Fogo, au-delà du cap Freels; cette île fut alors ajoutée sur les cartes avec le fleuve das Gamas (des Daines, correspondant au fleuve Gander), malgré les dangers que ces parages présentaient à la navigation. Le prototype Freducci illustre cette étape de l'évolution.

Il convient donc de restreindre considérablement l'étendue de l'exploration de Gaspar Corte-Real, au moins de celle dont il a pu communiquer la nouvelle au Portugal. On peut accorder qu'il s'est aventuré dans la baie de Bonavista, de navigation difficile, et qu'il a aperçu le cap Freels. Mais la baie Trinité a été le centre de son intérêt. Il n'a certes pas manqué de voir la baie de la Conception et le cap Saint-François. Mais il est

$\overline{\mathrm{du} \text { nord, }}$ qu'on achetait alors aux Flamands, aux Scandinaves et aux Anglais. On la pêchait dans la mer du Nord et en Islande.

123 La découverte de Terre-Neuve a eu une influence considérable sur le commerce de la morue, où, en peu d'années, les Français acquièrent une situation dominante. C'est apparemment aussi de cette époque que la morue devient l'une des principales sources d'alimentation des Portugais. de registre.

124 Biggar, Les Précurseurs, 96. Le mot est employé dans une entrée 
douteux qu'il soit descendu au sud jusqu'au cap de Raze. La carte Kunstmann III ajoute en effet le sud de la péninsule d'Avalon à un dessin plus ancien de Terre-Neuve reproduit par Kunstmann II et Kunstmann IV. Quant à ce qu'il a pu voir après le départ des vaisseaux envoyés par lui à Lisbonne, au début de l'automne de 1501, on ne le saura jamais, puisqu'il s'est perdu et n'a jamais été retrouvé. Les pêcheurs qui l'ont suivi ont après lui étendu et consolidé l'emprise du Portugal sur toute la côte, entre le cap Freels et le cap de Raze.

\section{L'EXPLORATION DE FAGUNDES}

L'événement qui a modifié l'étendue du littoral revendiqué par les cartographes portugais à Terre-Neuve est sûrement l'exploration de Jean Alvares Fagundes. On sait que cet explorateur était un gentilhomme originaire de Viana, au nord du Portugal. On conserve l'acte d'une donation que le roi Manuel lui a faite, le 13 mars 1521, de terres et d'îles précédemment découvertes par lui à proximité des possessions des Corte-Real. Fagundes aurait même tenté d'établir une colonie dans son domaine, après 1521, tentative qui aurait abouti à un échec.

Quelques historiens, par exemple Harrisse ${ }^{125}$ et Ganong ${ }^{126}$, ont essayé de reconnaître les vestiges de Fagundes sur les cartes du seizième siècle. Il ne nous semble pas qu'ils l'aient fait avec succès. Il est certain que l'exploration de ce découvreur a eu une influence sur la cartographie portugaise. Le seul nom des Onze-mille-Vierges, si caractéristique de la toponymie fagundésienne et si souvent attaché au littoral sud de Terre-Neuve, suffirait à le démontrer. Mais aucun cartographe ne s'est donné comme tâche de rapporter au complet ces découvertes, en situant chacun des noms créés par l'explorateur sur les lieux qui leur convenaient. L'expédition a généralement eu pour conséquence d'agrandir le domaine auquel le Portugal

125 Henry Harrisse, Découverte et évolution cartographique de TerreNeuve (Paris, London, 1900), 214-226.

126 W. F. Ganong, "Crucial maps...", Mémoires de la Société Royale du Canada (1930), II: 135-187. Ganong situe l'exploration de Fagundes partie sur la côte méridionale de Terre-Neuve, partie sur l'île du CapBreton et en Nouvelle-Ecosse. 
prétendra à Terre-Neuve. Toutes les cartes qui allongent la côte orientale de ce pays au nord de l'île Fogo et qui déposent des noms sur le littoral méridional à l'ouest du cap de Raze en porte des traces. Mais le découvreur lui-même a été moins remarqué qu'on ne croirait. On s'est moins occupé de signaler les possessions acquises par lui que de définir l'aire où le Portugal, grâce à lui, avait conquis ses droits.

Voici l'extrait le plus précieux qui se rapporte aux découvertes de Fagundes; il énumère les terres qui vont lui appartenir.

La terre qu'on dit être ferme qui se trouve depuis la démarcation de Castille - attenante du côté du sud à nos frontières - jusqu'à la limite de la terre que les Corte-Real ont découverte; les trois îles dans la baie de l'Aiguade, à la côte du nord-estsud-ouest; et les îles auxquelles lui, Fagundes, a donné des noms, à savoir Saint-Jean et Saint-Pierre, et Sainte-Anne et Saint-Antoine; et les îles de l'archipel de Saint-Pantaléon, avec l'île de Pitiguoem; et les îles de l'archipel des Onze-mille-Vierges; et l'île de Sainte-Croix, qui est au pied du banc, et une autre île qui s'appelle aussi de Sainte-Anne, qui a été vue mais dont on n'a pas pris possession ${ }^{127}$.

Ce témoignage se trouve quelque peu précisé par un autre, de beaucoup postérieur, qui paraît se rapporter au même sujet.

Il y a environ 45 ou 50 ans (en 1570), certains gentilshommes de Viana s'associèrent et, à cause de quelque information qu'ils avaient eue de la terre neuve de Bacalhao, ils décidèrent d'aller en habiter quelque partie, comme ils y allèrent en fait dans une nef et une caravelle. Et parce qu'ils trouvèrent très froide la terre où ils avaient d'abord décidé de s'ar-

\footnotetext{
127 "a terra que sse diz ser ffirme, que he des a demarcaçam de Castella que parte de banda do sull com a nossa demarcaçam, atee viir partir com a terra que os Corte rreaes deescobrirom, que hee da banda do norte; aas tres ilhas na baya d'auguoada, na costa de nordeste e sudueste; e as ilhas a que elle pos nome ffagumdas sam estas, a saber: sam Joam e sam Pedro, e santa Ana e santo Antonio; e as ilhas do arçepelleguo de sam Panteliom com a ilha de Pitiguoem, e as ilhas do arçepelleguo das honze mill virgeens; $\mathbf{E}$ a ihla de santa Cruz, que esta no pee do banco; e outra ilha que sse chama tanbem de santa Ana, que foy vista e non apadroada" (Biggar, Les Précurseurs, 128).
} 
rêter, ils coururent le long de la côte est-ouest, jusqu'à arriver à la côte du nord-est-sud-ouest. Et c'est là qu'ils habitèrent... Et cela se trouve au cap de Breton, lieu à l'entrée de la côte qui court au nord en une belle baie, où il y a beaucoup de monde et, dans la terre, des choses de grand prix ...128.

Dégageons de ces textes ce qui donne lieu à une interprétation certaine. On sait que le nom des Onze-mille-Vierges se trouve posé sur un groupe d'îles à l'angle sud-ouest d'une pointe, sur la rive méridionale de Terre-Neuve. Il n'y a que l'archipel de Saint-Pierre-et-Miquelon qui corresponde à cette situation et il n'est pas douteux qu'il soit représenté sous le nom des Onzemille-Vierges. Lorsque le nom usuel de Saint-Pierre est apparu sur les cartes françaises, après $1540^{129}$, celui des Onzemille-Vierges, déjà corrompu en Virgens sur quelques cartes, était trop connu pour disparaître tout à fait. Il a émigré vers l'ouest, sur la moitié du littoral sud de Terre-Neuve qui venait alors seulement d'apparaître dans la cartographie et qui n'avait aucune toponymie ${ }^{130}$. C'est ainsi que Virgens, ou Virgines, a pu durer jusqu'à nos jours, très défiguré, comme le nom d'un banc de morue et d'un groupe de petites îles: Burgeo. Il est donc assuré que l'exploration de Fagundes s'est faite en partie le long de la côte sud de Terre-Neuve.

128 "Haverá 45 annos ou 50, que de Viana se ajuntarão certos homen's fidalgos, e'pola informacão que tiverão da terra Nova do Bacalhao, se determinarão a hir povoar algûa parte della, como de feito forâo em hũa nao e hũa caravella, et por acharem a terra muito fria, donde hião determinados, correrão para a costa de leste Oeste, té darem na de nordeste sudoeste, e ahi habitarão... e isto he no Cabo do Britão, logo na entrada da Costa, que corre ao norte em hũa fermoza bahia, donde tem grande provoaçao e ha na terra cousas de muito preço..." (Biggar, Les Précurseurs, 195).

129 L'île de Saint-Pierre est mentionnée, pour la première fois à notre connaissance, sur la carte harléienne (Biggar, The Voyages of Jacques Cartier, pl. VIII, après la p. 128). Elle est alors située près du cap Breton actuel, tandis que les 11000 Vierges conservent leur site déjà traditionnel sur Saint-Pierre-et-Miquelon. Mais la carte Vallard, de 1547, remplace les 11000 Vierges par l'appellation de Saint-Pierre, sur le site véritable. De même, la mappemonde Desceliers de 1550 et la carte Le Testu de 1555. Sur cette dernière, l'île "aux Vierges" est sur la moitié ouest de la côte méridionale de Terre-Neuve, à l'endroit où se trouve aujourd'hui Burgeo.

${ }^{130} \mathrm{La}$ plus ancienne représentation de cette partie de Terre-Neuve est celle de Desceliers, en 1546. La mappemonde Vallard, de 1547, inaugure la représentation de cette partie comme une île en forme de croissant. 
Mais il est impossible de placer tous les noms de Fagundes sur cette même rive sud. Il faut chercher où mettre l'autre partie. Le second des textes cités suggère d'ailleurs un autre endroit: "Et parce qu'ils trouvèrent très froide la terre où ils avaient d'abord décidé de s'arrêter, ils coururent le long de la côte estouest, jusqu'à arriver à la côte du nord-est-sud-ouest." Les conceptions géographiques représentées ici sont celles de la première moitié du seizième siècle, qui ignorait encore l'existence du détroit de Cabot au sud de Terre-Neuve et l'insularité de cette dernière. La "côte est-ouest" est la rive méridionale, entre le cap de Raze et l'archipel de Saint-Pierre-et-Miquelon (Onzemille-Vierges). L'archipel lui-même est au coin est de l'entrée d'une baie qui incurve profondément le littoral vers le nord; et le côté ouest de la même baie est concédé aux Bretons, le coin opposé à l'archipel étant appelé cap des Bretons. De ce cap, la côte se prolonge sans interruption vers le sud-ouest, qu'elle soit la côte de Verrazano ou qu'elle soit celle de Gomez, selon les familles de cartes. Cette conception était celle de Pierre Crignon, qui faisait une seule côte continue du sud de Terre-Neuve et de la côte de Verrazano: "Poursuivant outre le cap des Bretons, il y a une terre contiguë audit cap, de laquelle la côte s'étend ponent et un quart de garbin (sud-ouest)..."131. La colonie de Fagundes s'est de la sorte établie à la frontière entre le territoire portugais et la terre des Bretons, sans doute sur le côté est de la baie, puisque l'ouest est territoire breton et souvent situé à l'ouest de la ligne de Tordesillas, que les Portugais n'entendent pas traverser. Exactement, son site se serait trouvé tout près de l'archipel des Onze-mille-Vierges, sur la péninsule de Burin, sinon sur l'archipel lui-même.

Mais cette colonie était une deuxième tentative de Fagundes. La première ne s'était pas faite sur la côte est-ouest, c'est-à-dire entre le cap de Raze et l'archipel. On ne pourrait la situer à l'ouest de la seconde colonie, parce que les colonisateurs sont venus de l'est à celle-ci: longer la côte est-ouest jusqu'à la côte sud-ouest-nord-ouest, c'est nécessairement venir de l'est et aller vers l'ouest. On ne peut non plus la situer plus

131 Ramusio, Navigationi et Viaggi, III : 423F. 
au sud que le deuxième établissement, parce que le froid excessif est ce qui a fait rater la première tentative; elle a donc eu lieu plus au nord. En sorte que Fagundes a dû faire un premier choix sur la côte orientale de Terre-Neuve, et plus au nord que l'île Fogo, s'il tenait à respecter la concession déjà faite aux Corte-Real.

On notera aussi que les explorations de Fagundes s'étendent entre deux méridiens. D'abord celui de la démarcation de Castille, la ligne de Tordesillas, limite occidentale qu'il ne doit pas traverser, s'il veut demeurer dans la zone d'expansion portugaise. Puis une autre longitude, qu'il voit plus à l'est comme la limite des terres concédées aux Corte-Real. Entre les deux, Fagundes voit des terres inoccupées qu'il demande pour luimême. Les noms qu'il aligne dans sa requête partent de la démarcation de Castille et vont vers l'est, ou vers l'autre limite.

Au nord de Terre-Neuve, on peut situer très facilement et sans aucune subtilité toute la première moitié de la liste de Fagundes, en obéissant à la suggestion des cartes produites entre 1520 et 1530. Nous avons vu que le plus récent Marco des cartes portugaises est placé au cap Dégrat. Voilà sans doute la ligne de démarcation. Et la "côte sud-ouest-nord-est" mentionnée dans l'acte de donation ne peut alors être autre chose que celle de Terre-Neuve depuis la baie Blanche jusqu'au même cap, laquelle a justement cette orientation. La baie de l'Aiguade, avec ses trois îles, coïnciderait très bien avec la baie de Saint-Lunaire, un havre particulièrement apprécié des marins français de l'époque. Descendant ensuite vers le sud en gardant la côte de Terre-Neuve en vue, Fagundes aperçoit deux grandes îles, les Belles-Isles des Français, qu'il appelle Saint-Jean et Saint-Pierre. On sait que les cartes portugaises, après 1520 , ne manquent pas souvent de signaler ce couple onomastique à cet endroit. ${ }^{132}$ Poursuivant toujours plus au sud, l'explorateur voit encore deux îles, non loin de la rive de Terre-Neuve. Il les nomme Sainte-Anne et Saint-Antoine. Elles sont aujourd'hui

$132 \mathrm{Ne}$ citons que les plus anciennes cartes à mentionner ce couple: Miller I, Pedro Reinel. 
appelées de Sainte-Barbe ou du Cheval. Les anciennes cartes portugaises n'ont pas retenu les noms fagundésiens de ces îles, parce qu'elles n'étaient pas sur la route des marins. L'orientation du littoral force maintenant Fagundes à se diriger vers le sud-est et à traverser la baie Notre-Dame. Il vient se heurter à une multitude d'îles de toutes grandeurs, qui lui barrent l'accès du rivage et dont la plus grande est l'île Fogo. Il donne à tout ce groupe le nom d'archipel de Saint-Pantaléon.

Mais le nom suivant, Pitiguoem, est capital pour la reconstitution du voyage de Fagundes. Ce nom n'a pas été inventé par l'explorateur; il l'a trouvé sur les lieux et il le signale comme déjà connu. L'île ainsi désignée est mise à part de l'archipel de Saint-Pantaléon, mais elle y est aussi reliée. À cause de la notoriété qu'on semble lui attribuer, Pitiguoem devrait aussi se retrouver sur les cartes; mais on ne l'y voit jamais. En fait, il ne peut être qu'une mauvaise lecture, paléographiquement facile à expliquer ${ }^{133}$, pour Pinguoem, Pingouin. Or l'île des Pingouins n'est qu'un autre nom pour l'île des Oiseaux, notre île Funk, qui était, nous l'avons montré, une étape nécessaire de la navigation vers le détroit de Belle-Isle. Le nom d'île des Oiseaux est fréquent sur les cartes et il a été repris par Jacques Cartier, en 1534. Mais celui de Pingouins a été employé concurremment avec lui. On le lit sur une carte portugaise dressée avant $1550^{134}$. Il est aussi mentionné dès 1536 par des explorateurs anglais qui se sont arrêtés à cette île ${ }^{135}$. Enfin, l'usage qu'en fait l'acte de donation de 1521 est certainement la plus ancienne de ces mentions. Le nom est d'autant plus remarquable qu'il n'était alors usité dans aucune des grandes langues européennes. C'est un mot breton: pen-gween, qui signifie têteblanche. Il a été créé sur notre île pour désigner le Plautus

133 Pour peu que le $n$ ait une forme irrégulière, il peut facilement se décomposer en deux lettres: it.

134 Carte Anonymo-Lopo Homem, dans Cortesão-Teixeira, Portugalix Monumenta cartographica, I: estampa 26. Le dessin du détroit qu'on voit sur cette carte a inspiré plusieurs cartes portugaises après 1545 , non moins que les cartes Homem, dont nous parlons plus loin.

${ }_{135}$ Dans un récit recueilli par Hakluyt de la bouche d'un membre de l'écuipage, Thomas Buts (Principall Navigations (London, 1589), 517-519). Le récit a ćté réédité par Biggar (The Voyages of Jacques Cartier, 274). 
impennis, espèce de pingouin aujourd'hui disparue à cause de la chasse immodérée que lui ont faite les pêcheurs ${ }^{136}$. Cet oiseau pullulait alors sur notre île. C'est là qu'a été forgé le mot pingouin qui sera adopté par le français au début du dixseptième siècle ${ }^{137}$ et qui passera aussi à plusieurs autres langues. Le portugais l'écrit aujourd'hui pingüim.

La présence de ce mot dans la description de Fagundes est très significative. Elle prouve d'abord, avec la mention de la "côte sud-ouest-nord-est" et celle des deux îles de Sam Joham et Sam Pedro, que notre reconstitution d'une première moitié de l'exploration au nord de Terre-Neuve est bien fondée. Elle confirme aussi que les Bretons ont précédé Fagundes dans ces parages, malgré les prétentions de découvreur que ce dernier affiche. Comme le nom pen-gween ne peut lui avoir été alors soufflé que par un Breton, il est probable même qu'il avait à son bord un pilote armoricain, qui l'a d'abord conduit en toute sûreté au cap Dégrat, où l'exploration a commencé. Le témoignage de Pierre Crignon, qui attribuait à des sujets français la découverte de cette partie de Terre-Neuve, se trouve encore une fois corroboré. Et l'on s'explique comment, vers 1520 , apparaît un nouveau groupe toponymique au nord de l'île Fogo. Les cartographes portugais ne paraissent pas immédiatement intéressés à l'expédition de Fagundes; mais ils rapportent les connaissances maritimes que les navigateurs de leur nation ont acquises de l'explorateur dans cette partie de l'océan. Enfin, se trouvent aussi confirmées nos conclusions, que l'extrémité nord de la côte de Corte-Real se trouvait au cap Freels. Fagundes se sent libre de faire des acquisitions au-delà de cette limite, entre le Marco qu'il pose plus à l'ouest sur le cap Dégrat et l'ancien Marco de Corte-Real, sur le cap Freels.

Aussi, l'exploration septentrionale de Fagundes s'arrêtet-elle à l'île des Pingouins. Après avoir longé vers le sud la côte de Corte-Real et s'être de nouveau avancé vers l'ouest

136 Voir une photographie de cet oiseau dans Biggar, The Voyages of Jacques Cartier, pl. II, après la p. 16.

137 Littré, Dictionnaire, 3: 1125. L'éminent linguiste ignore la préhistoire de ce mot, dont il ne retrace pas l'origine au-delà de 1600 . 
jusqu'à la ligne de démarcation de Castille, il recommence à l'archipel appelé par lui des Onze-mille-Vierges en direction de l'est. Il est difficile d'identifier avec certitude, voire avec probabilité, les deux îles que Fagundes nomme encore. Mais on ne doit pas oublier que l'explorateur se fait en même temps concéder la terre ferme entre les deux méridiens qui définissent la zone d'expansion portugaise, à l'ouest, et les possessions de la famille Corte-Real, à l'est. Il revendique donc la possession de toute la côte sud de Terre-Neuve entre l'archipel de Saint-Pierreet-Miquelon et le cap de Raze.

C'est pourquoi les noms portugais du sud de Terre-Neuve apparaissent sur les cartes en même temps que le détroit de Belle-Isle et la toponymie qui l'avoisine. La carte de Pedro Reinel ${ }^{138}$ est une importante exception à cette règle. Bien qu'elle rapporte la toponymie fagundésienne du nord, elle ne met aucun nom au sud de Terre-Neuve. Elle demeure en cela fidèle à une tradition cartographique des Portugais, qui connaissait bien les découvertes des Français au sud avant 1520, mais n'avait pas de raisons de leur faire une propagande. En effet, si jusqu'à cette date les pêcheurs portugais de Terre-Neuve semblent avoir ignoré le chemin de Belle-Isle, la côte méridionale de l'île était représentée sur les cartes, bien que sans toponymie. Cette représentation est encore très douteuse sur la carte de Contarini, de $1506{ }^{139}$. Mais elle est déjà probable sur la carte Ruysch, de 1507-1508 ${ }^{140}$, et sur la carte Maggiolo, de $1511^{141}$. En tout cas, elle ne fait plus de doute sur le prototype Freducci, qui est antérieur à Fagundes, c'est-à-dire à $1520^{142}$.

Sur les mêmes cartes portugaises où se manifeste l'influence de Fagundes apparaît aussi le nom des Bretons: cap des Bretens, terre des Bretons. Il reste peu de cartes de cette période qui se limitent au dessin de Terre-Neuve et n'y ajoutent pa 8.

138 Cortesão-Teixeira, Portugaliæ Monumenta Cartographica, I, estam-

139 Hoffman, Cabot to Cartier, 58, fig. 11.

140 Ibid., 66, fig. 15.

141 Ibid., 62, fig. 13.

142 Ibid., 70, fig. 17. 
pas les explorations de Gomez ou de Verrazano. Parmi elles, la carte de Pedro Reinel ne mentionne pas les Bretons, non plus que les autres noms de la côte méridionale. Mais leur nom apparaît sur la carte Miller I, qui est de la même catégorie ${ }^{\mathbf{1 4 3}}$. Ce même nom va devenir un trait constant des cartes de divers types qui rapportent ces deux explorations, à partir de 1525. Les savants qui ont étudié cette cartographie n'ont pas suffisamment remarqué un fait qui a des conséquences importantes pour une saine critique de telles cartes. Il existait déjà, avant Verrazano et Gomez, une représentation cartographique de Terre-Neuve qui avait sa propre valeur et à laquelle ces deux découvreurs n'ont rien ajouté. Le cap - ou terre - des Bretons était partie intégrante d'une telle représentation, mais non de la côte de Verrazano ou de celle de Gomez. Quand, après 1524, Vesconte de Maggiolo et Jérôme Verrazano voulurent représenter le littoral exploré par Jean Verrazano, ils le dessinèrent suivant les indications du découvreur, mais aussi ils l'achevèrent en le prolongeant au nord-est jusqu'au cap des Bretons des Portugais. Ainsi, la côte Verrazano et la côte portugaise ne furent plus qu'une seule côte continue de l'Amérique du Nord. Diogo Ribeiro usa d'un procédé identique, en reliant la côte de Gomez au cap des Bretons de la carte portugaise. Mais ni Verrazano ni Gomez n'avaient donné d'information sur la partie septentrionale du parcours qui leur était ainsi attribué, c'est-à-dire, en gros, sur ce qui est au nord de l'actuelle frontière canado-américaine. En sorte que, sur toutes ces cartes, la côte qui s'étend depuis la même frontière jusqu'au cap des Bretons est entièrement fictive et que la toponymie qui s'y trouve n'a absolument aucune valeur objective. Le cap des Bretons luimême ne doit pas être considéré comme l'extrémité nord-est de la côte Verrazano ou de celle de Gomez, mais bien comme l'extrémité sud-ouest de la côte connue par les Portugais avant les découvertes de ces explorateurs. Car il fait partie de la carte portugaise, non de la carte verrazanienne ou de la carte gomézienne. Il faut donc souligner que, jusqu'à Jacques Cartier, en 1534, la seule partie des cartes qui ait une valeur documenpa 24.

${ }^{143}$ Cortesão-Teixeira, Portugaliæ Monumenta Cartographica, I : estam- 
taire, en ce qui concerne le littoral canadien, est celle qui part du détroit de Belle-Isle, au nord, et, contournant l'est de TerreNeuve, a pour extrémité sud-ouest le cap des Bretons. En d'autres mots, la carte portugaise demeure, pour cette période, la seule qui ait valeur historique. Et elle ne comprend ni la moitié ouest du littoral sud de Terre-Neuve depuis la baie de Fortune jusqu'au cap de Raye, ni le détroit de Cabot, ni le reste du littoral canadien jusqu'à la frontière. Toutes ces parties qu'elle omet vont être graduellement révélées après 1534.

Dès avant 1520 , les pêcheurs portugais étaient au courant d'une occupation française au sud de Terre-Neuve et ce sont eux, sans doute, qui ont créé les expressions Terra dos Bretões et cabo dos Bretões pour désigner ce littoral. Les Français euxmêmes ne semblent pas avoir employé ces noms, sinon plus tard, à l'imitation des Portugais. Or, comme l'indique la toponymie qui a survécu, la terre des Bretons ne pouvait être que la section de côte comprise entre l'archipel de Saint-Pierre-et-Miquelon et le cap de Raze. C'est précisément cette côte méridionale que le prototype Freducci représente sans toponymie. Et c'est aussi celle que décrit Pierre Crignon. "Du cap de Ras au cap des Bretons, la côte court levant et ponent ... Entre le cap de Ras et le cap des Bretons habitent des peuples austères et cruels ... Sur cette côte, il y a d'excellentes pêcheries de molues, lesquels poissons sont pris seulement par les Français et les Bretons, parce que ceux du pays ne les prennent pas ... Cette terre a été découverte depuis 35 ans ençà, c'est-à-dire cette partie qui court levant et ponent, par les Bretons et les Normands. Pour cette raison, cette terre est appelée le cap des Bretons." Mais au-delà du cap des Bretons, on est hors du territoire autrefois découvert par les Bretons et les Normands: "Poursuivant outre le cap des Bretons, il y a une terre contiguë audit cap dont la côte s'étend ponent et un quart de garbin (sud-ouest) jusqu'à la terre de Floride ... laquelle côte a été découverte, il y a 15 ans, par messer Jean de Verrazzano au nom du roi François et de Madame la Régente..." ${ }^{144}$. Il est donc évident que ce qui est aujourd'hui,

144 Ramusio, Navigationi et Viaggi, III : 423. 
à partir du cap de Raze, la baie des Trépassés, le cap et la baie de Sainte-Marie, la baie de Plaisance, la péninsule de Burin et l'archipel de Saint-Pierre-et-Miquelon, tout cela appartient, dans la pensée de Crignon, à la terre appelée Cap des Bretons. Le cap des Bretons étant la borne occidentale de cette terre des Bretons qui se trouve sur Terre-Neuve ne peut que s'y trouver lui aussi. Dans la mesure où il a pu désigner un lieu précis, ce ne pouvait être que la péninsule de Burin. Car là s'arrête, à l'ouest, toute toponymie française antérieure à Cartier et c'est là que, venant du golfe, le découvreur rencontrera pour la première fois des signes d'occupation française encore en $1536{ }^{145}$.

En fait, cependant, le nom des Bretons n'apparaît sur les cartes qu'après 1520. Le roi Manuel ayant, le 13 mars 1521, concédé à Jean Alvares Fagundes le nord et le sud de TerreNeuve, les cartographes officiels étaient tenus d'indiquer l'étendue des terres revendiquées par leur pays. D'où l'addition d'une nouvelle section de côte au nord de la terre de Corte-Real et l'apparition d'une toponymie portugaise sur la côte méridionale, la ligne de Tordesillas passant désormais à l'ouest de SaintPierre-et-Miquelon. C'est pour qu'il n'y eût pas de confusion avec le littoral de Fagundes qu'on a transporté et le cap et la terre des Bretons hors de la zone portugaise, au-delà de la ligne de Tordesillas, sur une côte qui se trouvait là bien à propos. Mais comme il était déjà de tradition que le cap des Bretons se trouvait à la limite occidentale des terres connues, la terre des Bretons n'a jamais été mise, avant 1534, au sud-ouest du cap, mais bien au nord et au nord-est, ainsi que l'orientation de la côte le commandait. Ce n'est qu'après le transport du cap Breton au-delà du détroit de Cabot qu'il faudra bien se résigner à mettre la terre des Bretons au sud-ouest, car au nord et à l'est il n'y aura plus que l'océan.

Le pays découvert par les Bretons n'est pas en son lieu originel sur les cartes portugaises postérieures à 1520, mais la côte où il se trouve n'est pas entièrement une création des cartographes. Le prototype Freducci montrait dans l'hémis-

145 Biggar, The Voyages of Jacques Cartier, 238-239. 
phère nord quatre découvertes distinctes à l'ouest de l'Europe. Il évitait de les réunir, parce qu'on ignorait encore quels rapports elles avaient entre elles. Voici ces quatre pays, du nord-est au sud-ouest: $1^{\circ}$ la côte sud de la terre du Labrador, ou Groenland; $2^{\circ}$ la terre de Corte-Real, côte nord-sud depuis Fogo jusqu'au cap de Raze et côte est-ouest depuis le même cap jusqu'à Saint-Pierre-et-Miquelon; $3^{\circ}$ une côte nord-sud sans nom et sans toponymie, dont l'extrémité nord est un peu au-dessus des îles de Saint-Pierre-et-Miquelon et dont le sud est beaucoup plus bas que le sud de Terre-Neuve; $4^{\circ}$ une dernière côte nordsud appelée La Florida. C'est le troisième littoral qui attire ici l'attention; comme il est sans nom, nous l'appellerons terre Freducci pour la commodité du discours. La terre Freducci est bien séparée de Terre-Neuve, mais elle est aussi assez rapprochée du côté ouest de cette île, qui est formé par un méridien situé à l'ouest de Saint-Pierre-et-Miquelon ${ }^{146}$. Comme la terre Freducci n'apparaissait pas sur les anciennes cartes et qu'on la voit ici pour la première fois, on ne peut croire qu'elle a été une découverte de Corte-Real. Mais comme la carte Freducci ne porte encore aucune trace de l'exploration de Fagundes, on ne peut dire non plus qu'elle est une découverte de cet explorateur. D'autre part, elle n'est pas sans représenter quelque découverte portugaise. Déjà le témoignage de la carte Freducci n'est pas à rejeter. Et la carte Castiglione, de $1525^{147}$, indique clairement une exploration portugaise à cet endroit. Toutes les cartes postérieures de Terre-Neuve vont aussi la reproduire.

Or de découverte d'une côte nord-sud, dans cette région, il n'a pu y en avoir que sur l'île du Cap-Breton, dont une section entre le cap Breton et le cap Percé a justement cette orientation. Cela paraît confirmé par le fait que la carte Pedro Reinel - et d'autres ensuite - met une île, appelée Sam Joham, au sud de cette côte. Ce ne pourrait être que l'île de Scatari, située aujourd'hui près de notre cap Breton. Il y a donc lieu de croire qu'entre 1510 et 1520, quelque pêcheur portugais s'est fourvoyé jusqu'à notre cap Breton, qu'il aurait ainsi vu le premier. Ce

${ }_{146}$ Voir la reproduction de Hoffman, Cabot to Cartier, 70, fig. 17.

147 Ibid., 126, fig. 37. 
rivage, cependant, n'a pas été fréquenté par la suite. Le sort de l'île mentionnée par Reinel en sera une indication éloquente. L'île Saint-Jean, attachée originellement à cette découverte, va se déplacer en tous sens sur les cartes jusqu'à ce qu'elle se fixe ultimement sur ce qui est aujourd'hui l'île du PrinceÉdouard, dans le golfe Saint-Laurent. La terre Freducci a été une découverte dont la signification première s'est perdue.

Nous avons parlé plus haut de la tendance des cartographes de ce temps à réunir en un tout les informations diverses qu'ils possédaient. Elle s'exerce bien ici. Après 1520, l'habitude devient générale d'unir le Groenland à Terre-Neuve, en ne laissant subsister que la seule interruption du détroit de Belle-Isle. Il en a été de même de la côte méridionale de Terre-Neuve et de la terre Freducci. On peut facilement suivre les étapes de cette dernière réunion. Sur la carte Pedro Reinel, la séparation est conservée en forme de détroit. Sur Miller I, le détroit devient un estuaire rempli d'îles. Sur la carte Castiglione de 1525, l'estuaire a déjà la forme d'une baie en demi-cercle, l'exploration des deux côtés étant marquée d'un trait fort et le fond demeurant inachevé. Sur la carte Verrazano de 1529, la séparation a laissé comme vestige un fleuve se jetant dans la baie. Mais Maggiolo de 1527 a résolument dessiné tout le contour de la baie, qui va être perpétuée sur les cartes jusqu'après 1534. À l'entrée de la baie, du côté de l'est, on voit l'archipel des Onze-mille-Vierges (Saint-Pierre-et-Miquelon). Sur le pourtour, une tononymie qui augmente avec le temps. Et sur le côté ouest est parfois posé le nom de la terre des Bretons, tandis qu'à l'angle ouest de la baie, opposé à l'archipel des Onze-mille-Vierges, on voit presque immanquablement le cap des Bretons. Ce côté ouest de la baie, tout comme la terre Freducci qui lui a donné naissance, descendait plus bas que le côté est. Ce trait est le plus souvent conservé sur les cartes postérieures ${ }^{148}$.

Voilà donc une baie dont le côté oriental est vrai, le littoral ouest de la péninsule de Burin, flanqué de l'archipel des Onzemille-Vierges. Mais le côté occidental, originellement terre Fre-

148 Ibid., 123-129, fig. 35-40. 
ducci et résultat d'une découverte véritable, est devenu fictif par son rattachement à Terre-Neuve et son attribution aux Bretons. Car il n'y a pas à cet endroit de côte semblable sur Terre-Neuve. La baie correspondrait en partie à la baie de Fortune, dont le côté oriental est bien la péninsule de Burin, mais dont le côté ouest est beaucoup plus court que l'autre et continue ensuite directement à l'ouest, non au sud-ouest. La présence de la terre Freducci, devenue littoral des Bretons, à cet endroit prouve que les cartographes portugais ne savaient rien de la moitié ouest de la côte méridionale de Terre-Neuve, depuis la baie de Fortune jusqu'au cap de Raye. Et en fait, même les Français qui venaient chaque année à l'île de SaintPierre et à la baie de Plaisance ne s'aventuraient pas à l'ouest de l'archipel. Car, en juin 1534, Jacques Cartier commence seulement à soupçonner l'existence d'un passage maritime au sud de Terre-Neuve ${ }^{149}$. Et lorsqu'il franchit le premier ce passage en 1536, venant du golfe, il ne trouve sur cette rive aucun vestige d'occupation française avant l'île de Saint-Pierre. De toute la toponymie qui se trouve aujourd'hui sur cette partie de Terre-Neuve, il n'est pas un seul nom, sans excepter celui du cap de Raye, qui remonte même à 1536. Le dessin de la moitié ouest n'apparaît pour la première fois qu'en 1546, sur la mappemonde Desceliers ${ }^{150}$. Le nord du détroit de Cabot, à l'ouest des îles de Saint-Pierre-et-Miquelon, n'existait donc pas pour les Européens, avant que ne fussent connues les explorations faites par Cartier en 1534 et 1535/1536. C'est pourquoi la terre des Bretons, rejetée dans cette région par les cartographes portugais, ne peut être qu'une terre fictive.

La terre des Bretons, littoral occupé et fréquenté annuellement par les Français depuis 1504, ne pouvait done se trouver ailleurs qu'au lieu déjà indiqué par le témoignage positif de Crignon et par celui de la toponymie la plus ancienne qui subsiste en terre canadienne avec la toponymie portugaise, c'est-àdire la nomenclature française établie entre l'archipel de SaintPierre-et-Miquelon et le cap de Raze. Comment le cap Breton,

${ }^{149}$ Biggar, The Voyages of Jacques Cartier, 34-35.

150 Ibid., pî. XII, après la p. 192. 
expression vulgarisée par les cartographes portugais au moment où le littoral français était transporté par eux à l'ouest de Saint-Pierre-et-Miquelon, a-t-il pu sauter finalement de la côte de Terre-Neuve au sud du détroit ? Ce fut d'abord une conséquence cartographique de la découverte du golfe Saint-Laurent en 1534. Nous avons vu plus haut comment c'est à un cartographe portugais qu'on doit le premier dessin du golfe SaintLaurent, dès avant la seconde expédition de Cartier de $1535 / 1536$. Ce dessin est celui qui a servi de modèle à la carte de Jean Roze et à la carte harléienne ${ }^{151}$. Or ce même cartographe, mis au courant du soupçon qu'avait conçu Cartier sur l'existence d'un passage au sud de Terre-Neuve, a de nouveau séparé la terre Freducci de la rive méridionale de Terre-Neuve. Il l'a même isolée de la côte de Gomez, à laquelle on l'avait soudée depuis 1525 . Il y a là une préoccupation scientifique qui est à l'honneur du cartographe. Mais il ne s'agit que d'une reconstitution cartographique, non du rapport d'une information nouvelle sur l'existence de l'île du cap Breton. Car le cartographe n'en sait pas davantage sur les deux rives du détroit que n'en savait Cartier en 1534. Les îles de La Madeleine sont pour lui une partie du continent et la moitié ouest de la côte sud de Terre-Neuve n'existe pas encore. Seul le souvenir du prototype Freducci peut justifier la représentation de cette côte nord-sud qu'il éloigne de Terre-Neuve pour faire place à un détroit. Le cap Breton, déjà situé sur la terre Freducci depuis plus de dix ans, passe naturellement avec elle au sud du détroit. Il est digne de remarque que le nom du cap Breton n'est aujourd'hui accompagné d'aucune nomenclature aussi ancienne que lui. Lui-même est déjà mentionné par les Portugais peu après 1520. Mais la toponymie de la Nouvelle-Écosse et de l'île du cap Breton est presque entièrement postérieure à 1600 . Les deux noms les plus anciens sont ceux de cap Saint-Laurent et d'île de Saint-Paul, qui dérivent d'appellations données par Cartier en $1536^{152}$. Mais ils ont eu le temps de se corrompre ou de se déplacer sur les cartes avant de se fixer sur les lieux où on

151 Ibid., pl. V et VIII.

152 Ibid., 238. 
les voit aujourd'hui. Cartier avait appelé le cap Saint-Laurent cap de Lorraine; et le nom de Saint-Paul avait été mis sur le cap Nord, non sur l'île qui l'avoisine. C'est dire qu'après 1536 il $\mathrm{y}$ a eu encore un temps où ce littoral est demeuré désert.

Le dernier événement notable de l'épopée portugaise dans notre pays a été l'exploration de Jean Alvares Fagundes. On vient de voir l'influence considérable qu'il a exercée sur la cartographie. Mais, à côté des découvreurs, il convient de signaler en terminant le rôle des cartographes. Pour l'historien, il est presque plus important que celui des premiers. Songeons que la plus grande partie de l'information qu'on possède sur les découvertes antérieures à Jacques Cartier nous vient de la cartographie portugaise. Et pas seulement cela. Nous l'avons vu, c'est un Portugais qui a dessiné la première carte du golfe SaintLaurent, peu après sa découverte. Les premiers cartographes français de notre pays, dont les œuvres paraissent au jour après 1540, sont étonnamment dépendants de la cartographie hispano-portugaise, au point qu'ils introduisent des vocables ibériques même sur les rives du Saint-Laurent. Pour tout le littoral atlantique au sud du détroit de Cabot, ils ne produiront rien d'original durant le seizième siècle. Encore une fois, les premiers cartographes à donner le dessin véritable de la côte orientale du Canada furent des Portugais, les cartographes Homem. Pour les deux rives immédiates du détroit de Cabot, ceux-ci paraissent avoir été devancés par l'auteur de la carte Vallard, de 1547, qui dépendait lui aussi de sources portugaises ${ }^{153}$. Cette carte montre en effet une information autorisée sur la moitié ouest de Terre-Neuve et sur le nord de l'île du cap Breton, qui cesse de rappeler la terre Freducci ${ }^{154}$. Mais les Homem, Lopo et Diogo, donnent la première représentation

153 Elle n'a cependant pas de mérite spécial. Elle est l'une des cartes inspirées par le dessin portugais du détroit dont nous avons parlé à la note 134. "O atlas Vallard foi muito provàvelmente traçado por um cartografo portugerês residente na França, possivelmente o mesmo que, algunos anos anteo, delineara o atlas de Haia" (Cortesão-Teixeira, Portugaliæ Monumenta cartographica, V: 138).

${ }^{154}$ Le cap Breton s'y retrouve de nouveau soudé à la côte Gomez. 
véridique de toute la côte de la Nouvelle-Écosse et de la baie de Fundy sur leurs cartes de 1554 et de $1558{ }^{155}$.

Et l'occupation dont ils témoignent est française, comme l'atteste le fond de la toponymie superficiellement déformée par les rapporteurs portugais. Il y a lieu de supposer qu'avant 1550, les pêcheurs français de la baie de Plaisance se sont enfin aventurés au-delà du détroit et le long de la Nouvelle-Écosse. Un indice qui paraît confirmer cette supposition est mentionné par le P. Biard en $1616^{156}$. Les Souriquois, d'après ce missionnaire, donnaient à Terre-Neuve le nom de Présentic. Sur des lèvres indiennes Présentic était certainement Plesentig, qui est le nom de Plaisance à la forme locative. Ce nom leur a été enseigné par des pêcheurs français venant de la baie de Plaisance. D'autre part, nous nous sommes souvent demandé d'où venait l'appellation de Souriquois, donnée par les Français aux Micmacs dès avant le dix-septième siècle. L'explication la plus naturelle nous paraît être fournie par le lieu où les Français ont eu leurs premiers contacts avec ces Indiens. Vers 1550, on voit apparaître le nom de Xoracade sur les cartes Homem. Il est placé à proximité de notre cap Breton. Or Xoracade ne peut correspondre qu'à Solagadig (pr. Soulakadik), qui est le nom micmac de la baie de Miré, voisine du même cap. Les Français rendaient ordinairement le 1 micmac par un $r$. Ainsi, ils ont dû former le nom des Indiens de celui de ce lieu: Souriquois. Les relations suivies entre les pêcheurs français et les Micmacs ont probablement commencé à la baie de Miré entre 1540 et 1550 .

LuCIEN Campeau, S.J.

\footnotetext{
155 Voir les reproductions dans Ganong, "Crucial maps ...", Mémoires de la Société Royale du Canada (1930), II: fig. 24-29; aussi CortesãoTeixeira, Portugaliz Monumenta Cartographica, I: estampa 27. Ganong a, sans fondement, voulu voir un rapport entre ces cartes et l'expédition de Fagundes. Il y a bien une I. Fagunta sur la carte de Lopo Homem, de 1554. Mais le nom est disparu de celle de Diogo, en 1558. Ces cartographes ne daignent pas mentionner les Onze-mille-Vierges sur Terre-Neuve et ne témoignent aucune attention à la toponymie fagundésienne.

156 Le P. Pierre Biard au P. Claude Aquaviva, Port-Royal, 31 janvier 1612, Thwaites, The Jesuit Relations and Allied Documents, 2: 66. Cette édition donne la leçon Præsentis, mais l'original autographe du P. Biard a bien Presentic.
} 